\title{
X-ray source populations in the Galactic plane
}

\author{
A. D. P. Hands, ${ }^{1}$ R. S. Warwick, ${ }^{1 \star}$ M. G. Watson ${ }^{1}$ and D. J. Helfand ${ }^{2}$ \\ ${ }^{1}$ Department of Physics and Astronomy, University of Leicester, University Road, Leicester, LE1 7RH \\ ${ }^{2}$ Columbia Astrophysics Laboratory, Columbia University, 550 West 120th Street, New York, NY 10027, USA
}

Accepted 2004 February 27. Received 2004 February 25; in original form 2003 November 21

\begin{abstract}
We present the first results from the XMM-Newton Galactic Plane Survey (XGPS). In the first phase of the programme, 22 pointings were used to cover a region of approximately $3 \mathrm{deg}^{2}$ between $19^{\circ}$ and $22^{\circ}$ in Galactic longitude and $\pm 0.6^{\circ}$ in latitude. In total we have resolved over 400 point $\mathrm{X}$-ray sources, at $\geqslant 5 \sigma$ significance, down to a flux limit of $\sim 2 \times$ $10^{-14} \mathrm{erg} \mathrm{s}^{-1} \mathrm{~cm}^{-2}(2-10 \mathrm{keV})$. The sources exhibit a very wide range of spectral hardness, with interstellar absorption identified as a major influence. The source populations detected in the soft $(0.4-2 \mathrm{keV})$ band and hard $(2-6 \mathrm{keV})$ band show surprisingly little overlap. The majority of the soft sources appear to be associated with relatively nearby stars with active stellar coronae, judging from their high coincidence with bright stellar counterparts.

The combination of the XGPS measurements in the hard X-ray band with the results from earlier surveys carried out by ASCA and Chandra reveals the form of the low-latitude X-ray source counts over 4 decades of flux. It appears that extragalactic sources dominate below $\sim 10^{-13} \mathrm{erg} \mathrm{s}^{-1} \mathrm{~cm}^{-2}(2-10 \mathrm{keV})$, with a predominantly Galactic source population present above this flux threshold. The nature of the faint Galactic population observed by XMMNewton remains uncertain, although cataclysmic variables and RS CVn systems may contribute substantially. XMM-Newton observes an enhanced surface brightness in the Galactic plane in the $2-6 \mathrm{keV}$ band associated with Galactic ridge X-ray emission (GRXE). The integrated contribution of Galactic sources plus the breakthrough of extragalactic signal accounts for up to 20 per cent of the observed surface brightness. The XGPS results are consistent with the picture suggested from a deep Chandra observation in the Galactic plane, namely that the bulk of the GRXE is truly diffuse.
\end{abstract}

Key words: surveys - Galaxy: general - X-rays: general.

\section{INTRODUCTION}

With the current generation of X-ray astronomy missions, we are for the first time able to carry out high sensitivity, coherent surveys of selected regions of the Galactic plane. In particular, the $X M M-$ Newton mirrors afford a large collecting area $\left(\sim 4650 \mathrm{~cm}^{-2}\right.$ total geometric area) with good spatial resolution (full width at half maximum, FWHM, $\sim 6$ arcsec and half energy width, HEW, $\sim 15$ arcsec on-axis) over a wide field of view (30-arcmin diameter). In combination with the EPIC CCD cameras, this provides an excellent facility for surveying sky regions subtending many square degrees down to relatively faint flux levels in both the hard $(>2 \mathrm{keV})$ and soft $(<2 \mathrm{keV}) \mathrm{X}$-ray bands.

The goal of the XMM-Newton Galactic Plane Survey (XGPS) is two-fold. The first objective is to study the properties of the Galactic $\mathrm{X}$-ray source population at intermediate flux levels (down to $\sim 2 \times$ $10^{-14} \mathrm{erg} \mathrm{s}^{-1} \mathrm{~cm}^{-2}$ in the $2-10 \mathrm{keV}$ band, but an order of magnitude

${ }^{\star}$ E-mail: rsw@star.le.ac.uk fainter in flux terms in the softer $0.4-2 \mathrm{keV}$ band). The second is to search for extended, low X-ray surface brightness features including variations in the underlying diffuse Galactic ridge X-ray emission (GRXE; Worrall et al. 1982; Warwick et al. 1985; Koyama et al. 1986; Yamauchi \& Koyama 1993; Kaneda et al. 1997; Valinia \& Marshall 1998).

The nature of the X-ray source population at high X-ray fluxes was established by early all-sky surveys and subsequent identification programmes, which revealed that the brightest sources in our Galaxy are predominantly X-ray binaries and supernova remnants. More sensitive surveys of the Galactic plane have since been made, including those made by ROSAT (Motch et al. 1991) and ASCA (Sugizaki et al. 2001) complemented by the serendipitous surveys carried out with the Einstein observatory (Hertz \& Grindlay 1984). Together, these surveys have provided some glimpses of the X-ray source population at lower X-ray fluxes, and hence effectively at lower X-ray luminosities for Galactic objects, although the picture is far from complete. At soft X-ray energies $(<2 \mathrm{keV})$ ROSAT studies in particular have shown that coronal emission from relatively 
Table 1. Observation log for the XGPS-I.

\begin{tabular}{|c|c|c|c|c|c|c|c|}
\hline \multirow{2}{*}{$\begin{array}{l}\text { Field } \\
\text { Name }\end{array}$} & \multirow{2}{*}{$\begin{array}{c}\text { Observation } \\
\text { Date }\end{array}$} & \multicolumn{2}{|c|}{ Field Centre (J2000) } & \multirow{2}{*}{$\begin{array}{c}\text { MOS } \\
\text { exposure }^{a}\end{array}$} & \multirow{2}{*}{$\underset{\text { exposure }^{b}}{\text { pn }}$} & \multirow{2}{*}{$\begin{array}{c}\text { M OS } \\
\text { fraction }^{c}\end{array}$} & \multirow{2}{*}{$\begin{array}{c}\text { pn } \\
\text { fraction }\end{array}$} \\
\hline & & $\begin{array}{l}\text { R.A. } \\
(\mathrm{h} \mathrm{ms})\end{array}$ & $\begin{array}{l}\text { Dec. } \\
\left({ }^{\circ}{ }^{\prime \prime}\right)\end{array}$ & & & & \\
\hline Ridge 1 & $2000-10-08$ & 182600.4 & -121455.9 & 8393 & 5914 & 0.95 & 0.85 \\
\hline Ridge 2 & 2002-09-21 & 182648.4 & -115248.7 & 13667 & 12046 & 1.00 & 1.00 \\
\hline Ridge 3 & $2000-10-11$ & 182736.4 & -113040.4 & 12044 & 9648 & 0.95 & 0.85 \\
\hline Ridge 4 & $2000-10-12$ & 182817.0 & -110753.0 & 9256 & 12998 & 0.94 & 0.42 \\
\hline Ridge 5 & 2002-09-17 & 182906.0 & -104503.0 & 13667 & 12046 & 0.98 & 0.93 \\
\hline XGPS 1 & 2001-03-08 & 182504.6 & -115000.4 & 7794 & 4797 & 1.00 & 1.00 \\
\hline XGPS 2 & 2001-03-10 & 182734.0 & -120920.0 & 9144 & - & 1.00 & - \\
\hline XGPS 3 & 2001-03-10 & 182549.0 & -112842.7 & 9144 & - & 1.00 & - \\
\hline XGPS 4 & 2001-03-10 & 182819.4 & -114805.2 & 9144 & - & 1.00 & - \\
\hline XGPS 5 & 2001-03-22 & 182635.7 & -110732.1 & 9994 & 7348 & 0.96 & 0.95 \\
\hline XGPS 6 & $2001-03-22$ & 182905.9 & -112657.4 & 8794 & 6148 & 0.44 & 0.23 \\
\hline XGPS 7 & 2001-03-24 & 182721.2 & -104617.8 & 7637 & 4998 & 0.95 & 0.98 \\
\hline XGPS 8 & $2002-09-29$ & 182950.8 & -110541.2 & 13167 & 11546 & 1.00 & 1.00 \\
\hline XGPS 9 & 2001-03-26 & 182806.4 & -102510.0 & 11894 & 9248 & 0.92 & 0.62 \\
\hline XGPS 10 & $2001-10-03$ & 183036.2 & -104429.3 & 9767 & 8146 & 0.97 & 0.83 \\
\hline XGPS 11 & 2002-09-19 & 182943.9 & -102409.6 & 8409 & 6788 & 0.92 & 0.81 \\
\hline XGPS 12 & $2002-09-27$ & 182859.1 & -100650.9 & 7367 & 5746 & 1.00 & 1.00 \\
\hline XGPS 13 & 2003-04-10 & 183125.5 & -102432.5 & 6666 & 5047 & 0.73 & 0.66 \\
\hline XGPS 14 & 2002-03-11 & 183029.3 & -100247.1 & 7269 & 4998 & 1.00 & 1.00 \\
\hline XGPS 15 & 2002-03-27 & 182936.6 & -94241.1 & 7274 & 4998 & 0.82 & 0.96 \\
\hline XGPS 16 & $2002-03-15$ & 183206.5 & -100151.8 & 7762 & 5486 & 1.00 & 1.00 \\
\hline XGPS 17 & 2002-03-29 & 183113.9 & -94139.4 & 7274 & 4998 & 0.99 & 0.94 \\
\hline
\end{tabular}

Notes. ${ }^{a}$ Total exposure for the MOS 1 camera (s). ${ }^{b}$ Total exposure for the pn camera (s). ${ }^{c}$ Fraction of the exposure time used in producing images.

nearby active stars dominates (e.g. Motch et al. 1997). Above $2 \mathrm{keV}$ the characteristics of the harder population are far less well-defined, although it is clear that accreting binary sources (both X-ray binaries and cataclysmic variables) make a significant contribution.

To date, the XGPS survey has been targeted at several locations in the Galactic segment between the Galactic Centre and the Scutum spiral arm. Here we report the results from the first phase of the XGPS (hereafter XGPS-I), which has entailed a total of 22 XMMNewton pointings, covering a region of approximately $3 \mathrm{deg}^{2}$ between $19^{\circ}-22^{\circ}$ in Galactic longitude and \pm 0.6 in latitude. Over 400 discrete point-like X-ray sources have been detected in XGPS-I and in this paper we focus on the properties of this source population and the contribution these discrete sources make to the GRXE. In a second paper (Hands et al., in preparation) we will present the results of a search for low-surface brightness, spatially extended Xray sources in the XGPS-I fields and also report on the properties of the underlying diffuse GRXE.

\section{OBSERVATIONS}

The XGPS-I programme comprises 22 XMM-Newton pointings carried out during the period between 2000 October and 2003 April (see Table 1). Five of these observations formed part of the SSC Guaranteed Time programme (the Ridge 1-5 fields), whereas the remaining time was awarded to an $\mathrm{AO} 1$ programme (PI: RSW; the XGPS 1-17 fields). The allocated exposure times for these two sets of observation were 9 and $5 \mathrm{ks}$, respectively, although in most instances somewhat longer exposure times were actually scheduled (see Table 1). In all cases the EPIC cameras were operated in Full Window Mode with the medium filter selected. In the event, the completion of this survey proved problematic due to the impact of intervals of high instrumental background on the data quality, at least for a subset of the pointings. ${ }^{1}$ Several of the pointings were in fact repeated so to mitigate the worst effects of this contamination (Table 1 refers to the observations actually used in the present work).

\section{DATA ANALYSIS}

\subsection{Data screening and image extraction}

We have analysed the X-ray data from the three EPIC cameras on $X M M-N e w t o n$, two of which incorporate MOS CCDs (MOS 1 and MOS 2; Turner et al. 2001) and one based on pn technology (Strüder et al. 2001). As noted earlier, the instrument background in both the MOS and pn cameras is highly variable with the transition from quiescent conditions to a severe flaring episode often occurring on time-scales shorter than $\sim 1000 \mathrm{~s}$ and involving an increase in the background count rate by factors ranging from a few up to several orders of magnitude. In order to assess the background conditions in each of the XGPS-I observations, we have extracted the full-field light curve for events with energy in the range $0.2-12 \mathrm{keV}$. The results are illustrated in Fig. 1 for the MOS 1 camera.

Prior to constructing X-ray images, the X-ray events recorded in each EPIC camera for each observation must be filtered in various ways. $^{2}$ The first step was to use the full-field light curve to exclude time-intervals when the instrument background was unduly high.

\footnotetext{
${ }^{1}$ The background enhancements are attributed to a highly variable flux of soft protons which in orbit appear to be channelled by the X-ray mirrors on to the CCD detectors.

${ }^{2}$ We take as a starting point in our analysis the calibrated event list produced by the standard $X M M$-Newton pipeline, together with exposure maps and other pipeline products. We select X-ray events corresponding to patterns $0-12$ for the MOS and $0-4$ in the pn.
} 

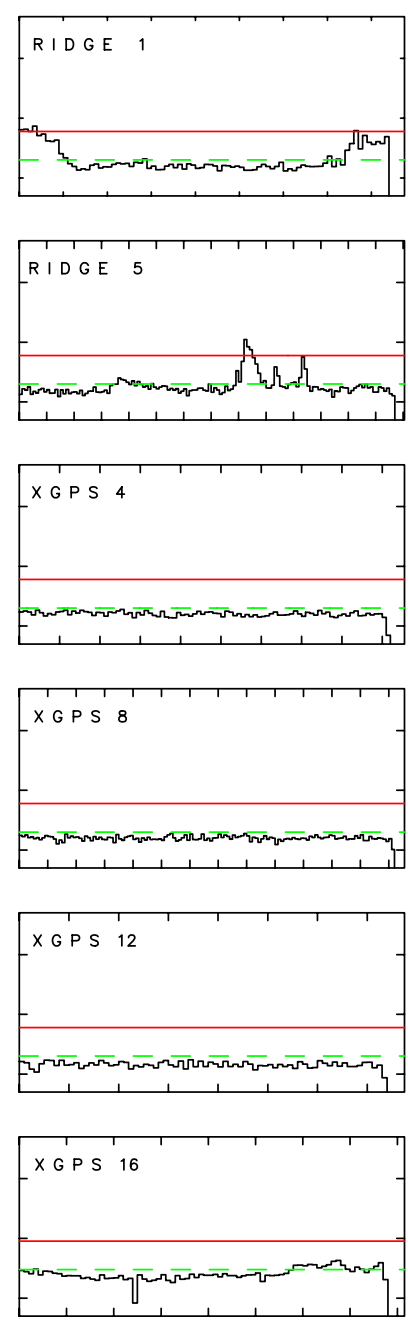
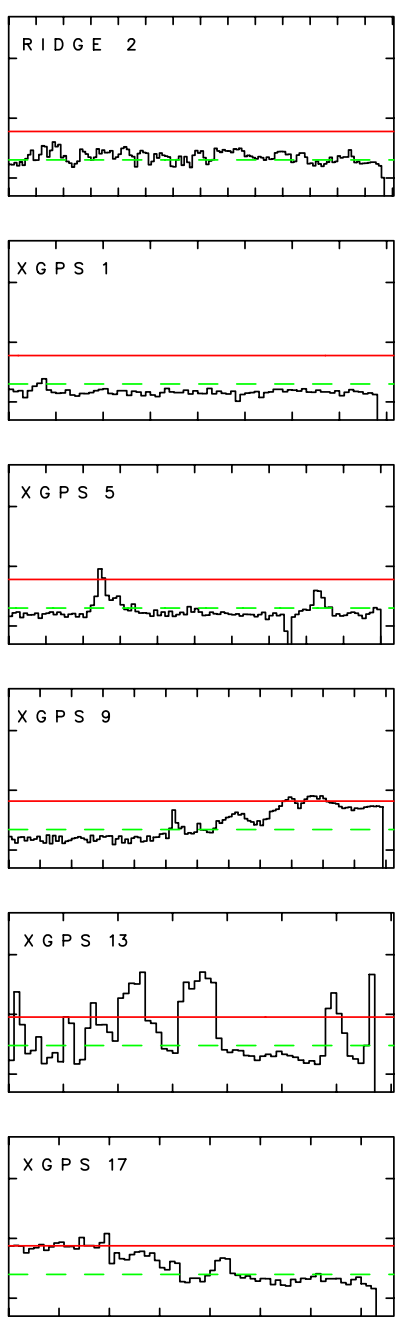
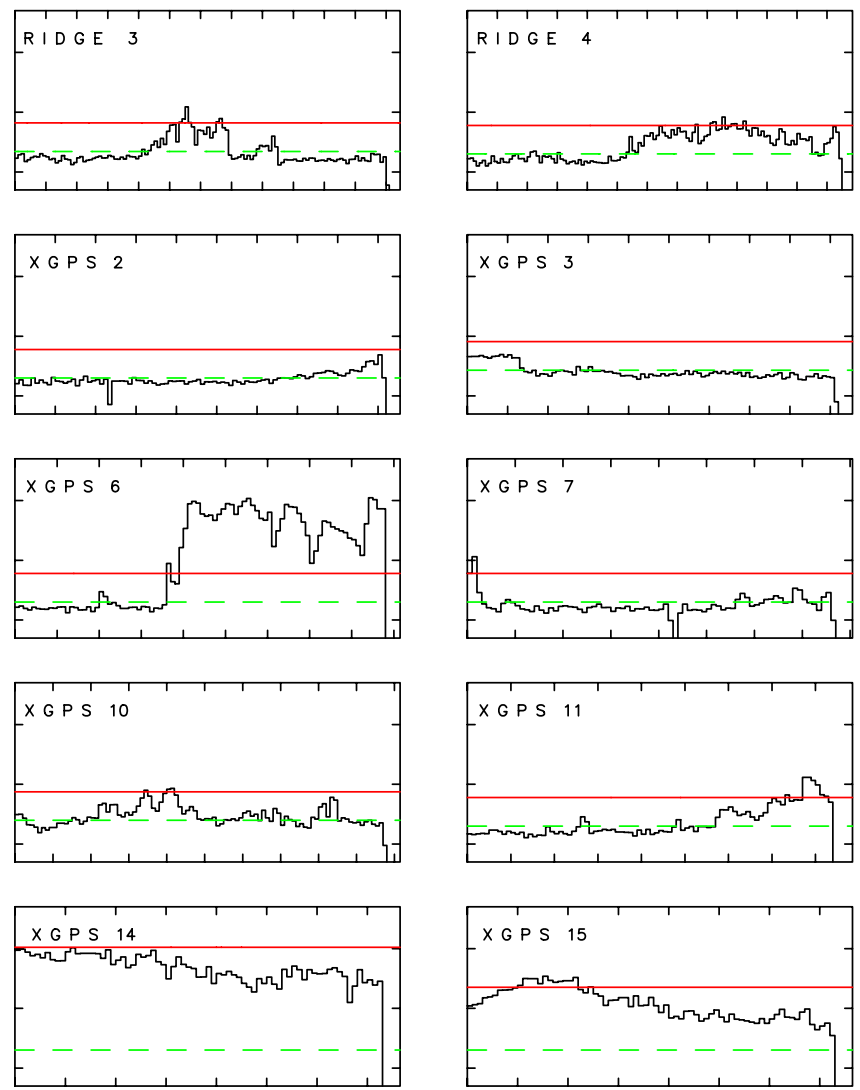

Figure 1. MOS 1 full-field light curves for all 22 XGPS-I observations. In each case the units of the vertical axis are counts per $100 \mathrm{~s}$ in the full $0.2-12 \mathrm{keV}$ band. The scaling is logarithmic with the three tick marks corresponding to 100, 1000 and 10000 counts per $100 \mathrm{~s}$, respectively. The unit of the horizontal axis is time with tick marks every $1000 \mathrm{~s}$. The thresholds used to exclude the most intense background flares are shown in each case (solid line). The more stringent thresholds used to extract data sets with 'near-quiescent' background conditions are also indicated (dashed line).

The fact that the XGPS-I observations are relatively short (i.e. 6$14 \mathrm{ks}$ actual exposure time) and the background flaring episodes are rather common and have durations of several ks or longer, means that 'near-quiescent' background conditions are typically experienced only for a fraction of the total on-time (with this fraction approaching zero in some observations). As a compromise between selecting clean data on the one hand and having sufficient source counts to make source detection effective on the other, we set a threshold for data exclusion at a full-field count rate roughly three times higher than the 'lowest level' of the observation (see Fig. 1 and Table 1). For most of the observations this resulted in a cut at a rather similar count-rate setting. However, the XGPS 14 and XGPS 15 observations were subject to enhanced background levels throughout the exposure and for these a significantly higher threshold count-rate was required (implying a much reduced sensitivity to faint cosmic X-ray sources in these observations). For some purposes (e.g. searching for low-surface brightness X-ray features within a particular observation or looking for variations in the underlying GRXE across many fields) a much more stringent rejection of high background intervals is required. The thresholds used to identify near-quiescent background conditions are also shown in Fig. 1.
All the data from the XGPS 14 and XGPS 15 observations were rejected for this analysis.

This empirical approach is broadly similar to that later adopted for the construction of the first XMM-Newton catalogue (Watson et al. 2003).

Once the temporal filter has been applied, the next step is to make images in specific energy bands for further analysis. Here we have used a soft $(0.4-2.0 \mathrm{keV})$ band, a hard $(2.0-6.0 \mathrm{keV})$ band and a broad-band representing the combination of the soft and hard channels (0.4-6 keV). Our choice of bands was made to optimize detection signal-to-noise ratio. Note that it differs somewhat from that used in the standard XMM-Newton data products. In the case of the MOS cameras we specifically excluded two narrow energy bands which are contaminated by fluorescent $\mathrm{Al}$ and $\mathrm{Si}$ lines originating within the detector (see Fig. 2 ). ${ }^{3}$ Although the Si line is not a prominent feature in the pn background, for consistency we use the same energy band selection for the pn data.

\footnotetext{
${ }^{3}$ The energy ranges excluded were $1.4-1.575 \mathrm{keV}$ and $1.675-1.8 \mathrm{keV}$ corresponding to the $\mathrm{K}_{\alpha}$ lines of neutral $\mathrm{Al}$ and $\mathrm{Si}$ respectively.
} 


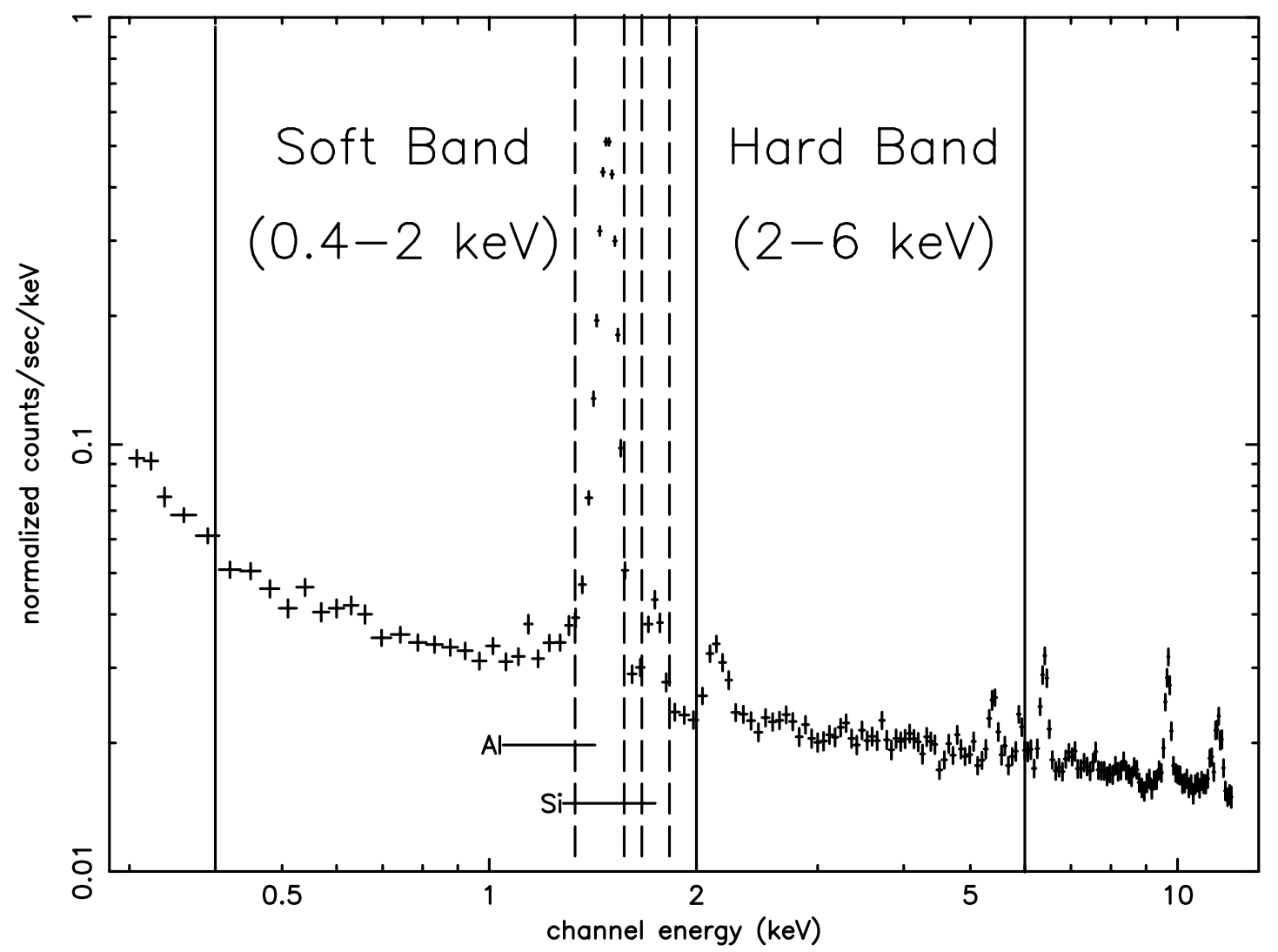

Figure 2. The spectra collected from edge regions of the MOS CCDs which lie underneath the filter wheel and hence are not exposed to the X-ray sky. The solid lines define the boundaries of the energy bands used in the analysis. The two regions identified by the dashed lines, which encompass the fluorescence lines of $\mathrm{Al}$ and Si produced within the MOS cameras, were specifically excluded from the soft band. Although other instrument fluorescence lines are present in the hard band, they are of relatively low intensity and have very little impact on the hard-band background count rates.

The final filter used to select the data is a spatial discriminator to distinguish between the areas of the CCDs which are exposed to the sky and those which are not. This is particularly important for the two MOS cameras for which a significant fraction of the area of the outer CCDs is shielded by the camera's filter wheel. The spatial mask used to perform this filtering was derived from a central band exposure map (2.0-4.5 keV), produced by the standard SAS pipeline procedure. In practice, we also applied additional masking so as to exclude the regions of the field of view where, due to the mirror vignetting, the effective exposure was less than 25 per cent of the on-axis value.

At this stage we were utilizing three energy bands (soft, hard and broad) per EPIC camera (MOS 1, MOS 2, pn), to give a total of nine separate X-ray images per observation, with each image consisting of a $600 \times 600$ array of 4 -arcsec pixels. In order to improve the signal-to-noise ratio we subsequently co-added each pair of MOS images. In carrying out source detection we have treated the MOS and pn data as completely separate channels, which can be compared for quality control purposes.

Fig. 3 illustrates the sky coverage of the survey for both the MOS and pn cameras in the form of a mosaic of the exposure maps from the individual XGPS-I pointings. In effect the survey uses three rows of pointings in a close-packed hexagonal pattern (with a spacing between adjacent field centres of 24 arcmin), so as to give efficient (but not particularly uniform) coverage of a narrow strip of the Galactic plane.

\subsection{Detecting sources}

We have employed an iterative sliding-box algorithm to detect point sources in the individual XGPS-I fields. ${ }^{4}$ Briefly, the process was as follows. The raw image was lightly smoothed and used to identify bright regions corresponding to individual point sources or groups of sources. These source regions were then excised and the remaining data heavily smoothed to produce a sky-background map. ${ }^{5}$ After subtracting the derived background map from the raw image, the data were again lightly smoothed and then scanned for local peaks above a specified surface brightness threshold. At the position of each local peak, we use a cell of radius 16 arcsec to extract a source plus background counts value (C) from the original raw image and a corresponding background estimate (B) from the background map. The net count from the source $(\mathrm{S})$ was then given simply as

\footnotetext{
${ }^{4}$ Our approach was developed before the standard SAS source detection chain was mature enough to be considered for this project. Although our procedures are simpler than those incorporated in the sAs chain used in standard $X M M-$ Newton processing, we do not expect that a detailed comparison would reveal any major differences in results and in particular our approach to background estimation is better-matched to the specific background issues faced at low Galactic latitudes and in low exposures.

${ }^{5}$ The light smoothing was performed using a circular Gaussian mask with $\sigma=8 \operatorname{arcsec}(1$ pixel $=4$ arcsec $)$, whereas the mask used for heavy smoothing was a square top-hat function of dimension $160 \mathrm{arcsec}$.
} 


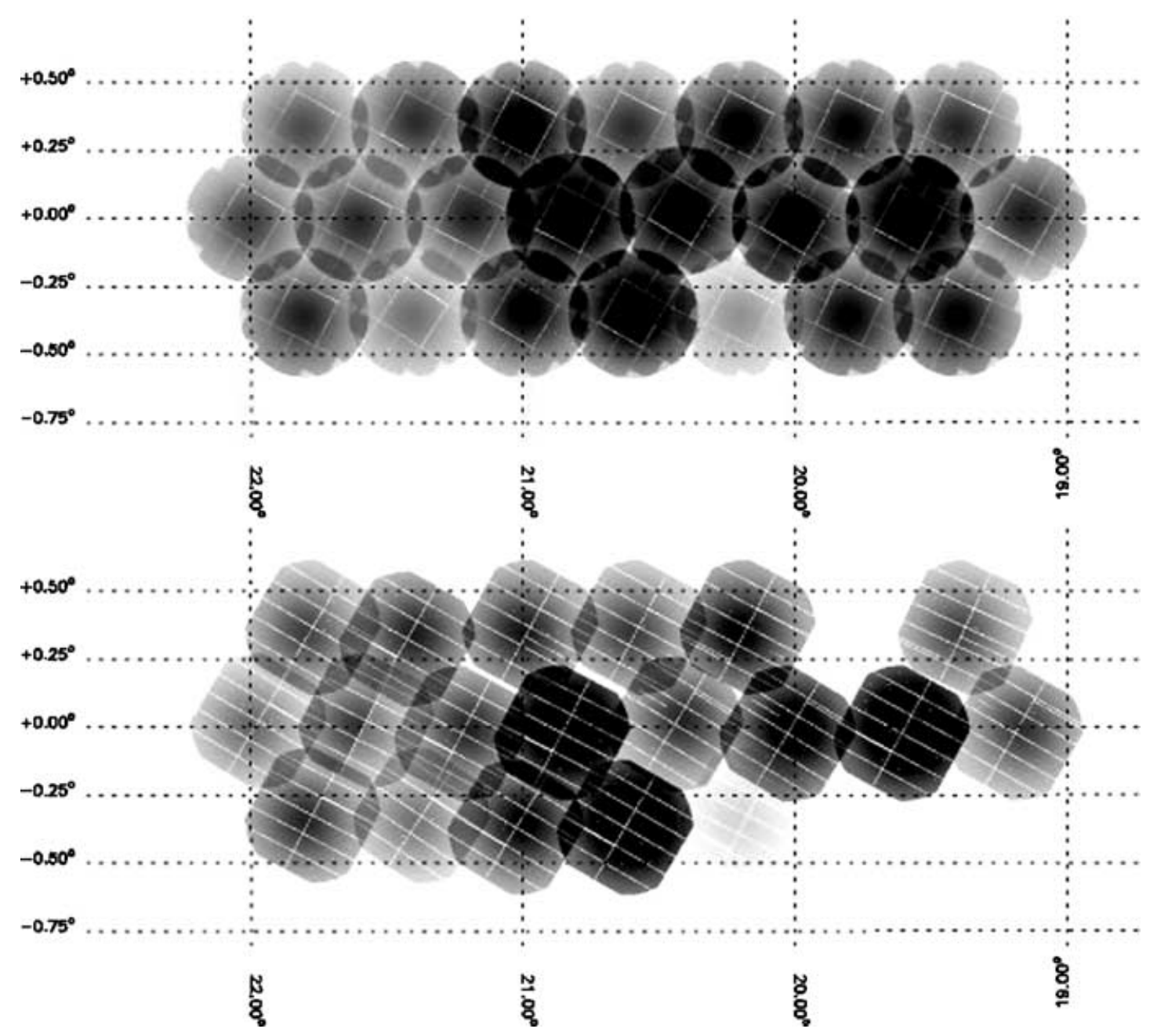

Figure 3. Top panel: a mosaicked (2-4.5 keV) exposure map showing the sky coverage provided by the MOS cameras over the full set of XGPS-I observations. The image is plotted in Galactic coordinates and employs a simple rectangular projection. The grey-scale (darker for longer exposures) corresponds to the accumulated (MOS $1+$ MOS 2) exposure time at different points in the survey region, with the variation for each individual pointing largely reflecting the vignetting function of the XMM-Newton mirrors. Bottom panel: the same information for the pn camera. The gaps in the pn exposure map correspond to XGPS-I observations for which pipeline-processed pn data are not available. The maximum exposure is 18 and $6.5 \mathrm{ks}$ in the MOS and pn images, respectively.

$S=C-B$. In order for a peak to qualify as a source detection, two criteria were applied, namely $S \geqslant 10$ and $S \geqslant 5 \sqrt{ } B$, implying a detection significance of $\geqslant 5 \sigma$. The list of sources detected by this method were subsequently used to define a new source mask and a revised background map. A further iteration of the source detection procedure then resulted in a final source list for the observation in question. Because the sensitivity to point sources depends on both the exposure time and the particle background rate, the sensitivity map of the full survey shows a somewhat different spatial variation to that of the corresponding exposure map (Fig. 4, top panel).

The process described above produces six separate source lists per XGPS-I observation (two detector channels and three spectral bandpasses). These source lists were merged by correlating all sources lying within 20 arcsec of each other. The position offsets for detections of the same source in two detector channels or in different bandpasses of the same detector were found to be distributed such that 68 per cent ( 90 per cent) were contained within a radius of $\approx 2.8 \operatorname{arcsec}(\approx 4 \operatorname{arcsec})$, which is consistent with the estimated statistical errors on the positions for sources at the faint end of the brightness distribution. However, a much broader correlation region was chosen so that sources would not be identified as distinct if the separation between them was less than or comparable to the half energy width of the EPIC point spread function. Fig. 5 shows a comparison of the MOS and pn broad-band detections in a central part of the Ridge 3 observation. In total four sources are detected in this subregion but interestingly only one of them is classed as a detection in both the MOS and pn cameras (see Section 4.1).

With the merging process for each observation completed, the next step was to select the best position information for each individual source and use this position to determine its flux. Here we used a quality parameter from the source detection process, representing the fraction of 'good pixels' in the source cell. This parameter is particularly useful in flagging sources near CCD chip gaps or distorted by bad columns in the CCD. The source position was taken from the (detector/spectral) channel with the highest quality value. Where more than one channel had quality $=1$ we arbitrarily used the priority sequence pn/broad, MOS/broad, pn/hard, MOS/hard, pn/soft, MOS/soft. At the assigned 'best position' we then extracted counts estimates $(\mathrm{S}=\mathrm{C}-\mathrm{B})$ using both a 16 arcsec and a 24 arcsec radius cell. The measured counts were subsequently corrected for the 


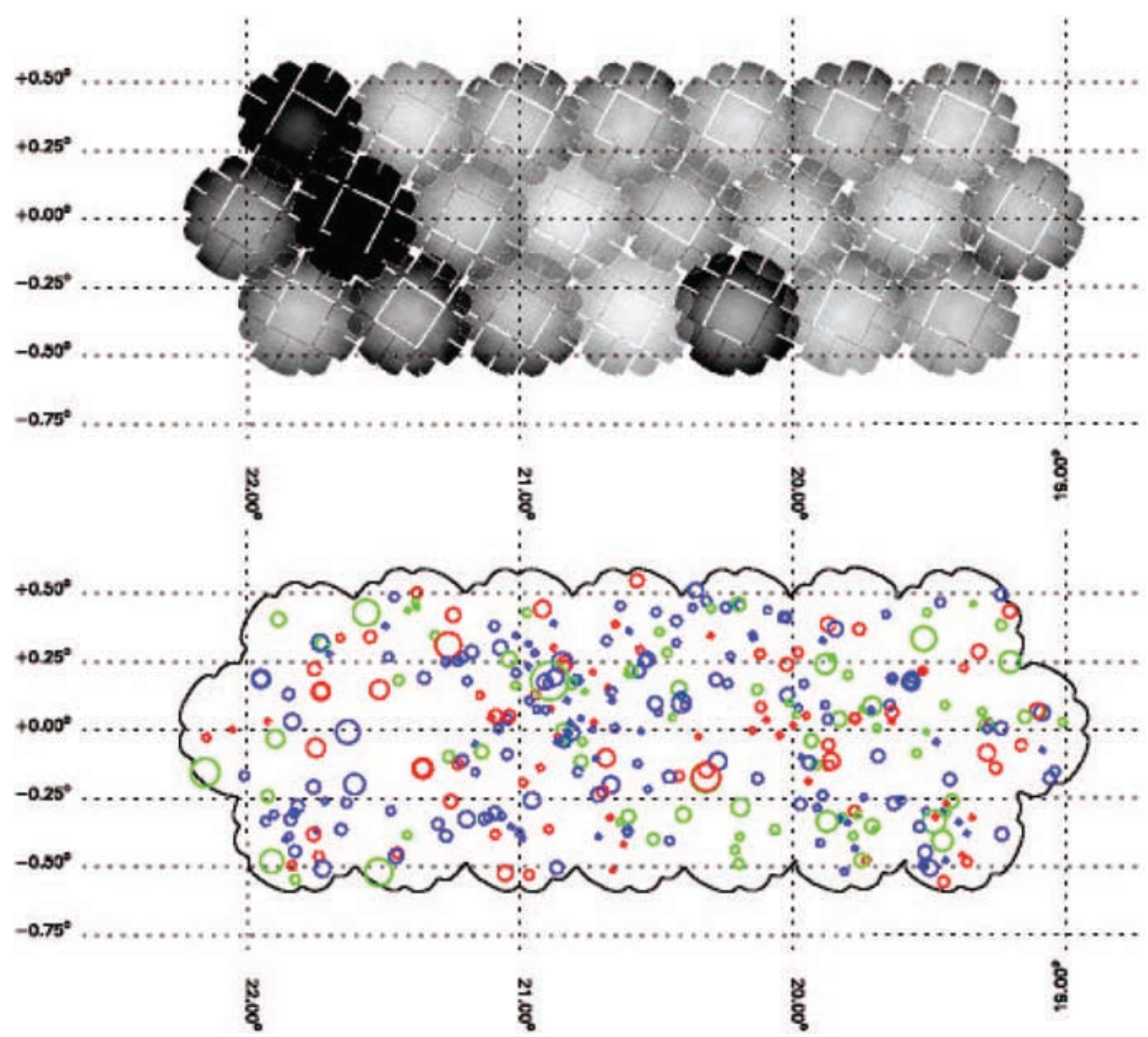

Figure 4. Top panel: mosaic showing the variation in source detection sensitivity across the 22 XGPS-I fields. The broad-band (0.4$6 \mathrm{keV}$ ) source count rate required to give a $5 \sigma$ detection varies from 0.5 count ks ${ }^{-1}$ (white) to 3 count ks $^{-1}$ (black) across the survey region dependent on the exposure time and particle background rate. Bottom panel: schematic image showing the sources detected by the MOS cameras in the soft ( $0.4-2 \mathrm{keV})$, hard (2-6 keV) and broad (0.4-6 keV) energy bands. Blue circles represent sources detected in the hard band but not the soft band; red circles represent sources detected in the soft band but not the hard band; green circles represent the remaining sources which are either detected in the soft and hard bands or in the broad-band alone. The radius of each circle is a logarithmic measure of the count rate of source.

signal loss outside the source cell and converted to on-axis count rates using appropriate exposure map information. ${ }^{6}$ We use the counts derived from the smaller cells in the source counts analysis, as these are matched to the source detection process and the derived sensitivity curves (see Section 4.3). For all other purposes we use the counts based on the 24 -arcsec radius cells.

The final step was to combine the source lists from the individual XGPS-I observations into one source catalogue. This involved the removal of a small number of duplicate entries where sources had been detected in more than one observation in regions where there was overlapping coverage; in practice preference was always given to the highest sensitivity detection.

\footnotetext{
${ }^{6}$ A source cell of radius $16 \operatorname{arcsec}$ (4 pixel) encompasses $\sim 70$ per cent of the counts from a point source (averaged over the field of view), whereas the corresponding value for a 24 arcsec (6 pixel) radius cell is 80 per cent. Unless otherwise stated, all the X-ray count rates quoted here are corrected for the signal loss due to the limited size of the source cell.
}

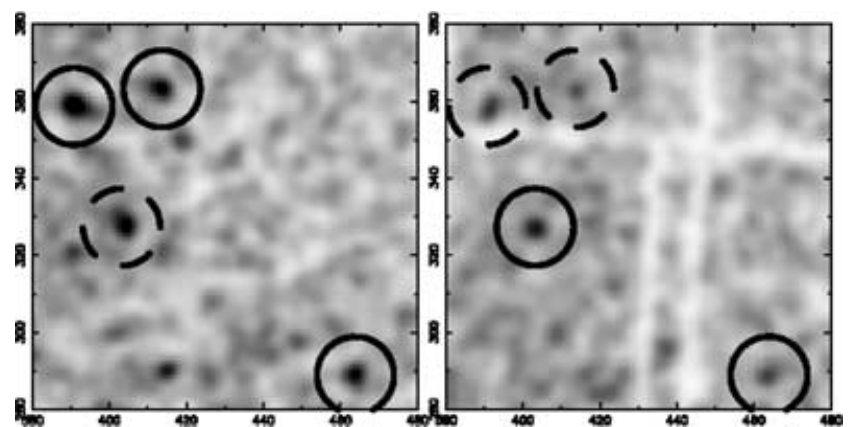

Figure 5. Lightly smoothed MOS (left) and pn (right) images of a part of the Ridge 3 field in the broad $(0.4-6 \mathrm{keV})$ band. The circles drawn with solid lines indicate local peaks (above a specified threshold) which meet the two source-detection criteria, whereas the circles drawn with dashed lines indicate peaks which failed this test. A total of four sources are detected in this subfield, but only one source is classed as a significant detection in both the MOS and pn cameras. 


\subsection{Extracting source spectra}

An algorithm was also developed to extract the net spectra of a defined set of sources in any particular field. In this case a more stringent version of the background rejection filtering was employed with the result that the two XGPS-I observations badly affected by background flaring were excluded (see Fig. 1). The source plus background events were accumulated from within circular regions of radius 24 arcsec centred on the identified source positions, with a similarly positioned array of annuli of radius 24-96 arcsec used to extract corresponding background events. Where two sources occur close to each other, the region of the annulus contaminated by the other source was excised. Background subtraction was carried out on a source-by-source basis and the resulting net-spectra summed over the defined set of sources prior to division by the effective exposure time. In effect this procedure provides the count-weighted average spectrum of the set of sources under consideration.

\section{RESULTS}

One of the main aims of the present survey is to study the X-ray source population of the Galaxy at relatively faint fluxes. In this section we consider the $\mathrm{X}$-ray source catalogue derived from the XGPS-I programme, the X-ray spectral properties of the sources and the source count statistics. We also give brief details of possible optical counterparts based on available wide-field optical data and other published catalogue information.

\subsection{The XGPS-I source catalogue}

A total of 424 discrete X-ray sources satisfied the detection criteria. Fig. 4 (bottom panel) illustrates the spatial distribution of the XGPS-I sources across the survey region. The full source catalogue, including the source positions and the measured count rates in both the MOS and pn cameras, is presented in Appendix A. The X-ray spectral hardness ratio (HR) quoted for each source is defined as:

$$
H R=\frac{H-S}{H+S}
$$

where $H$ is the number of counts measured in the hard band and $S$ is the corresponding number of soft counts. For sources detected in both camera systems we summed the MOS and pn counts in the two energy bands before calculating HR. A correction for the differential vignetting was not applied to the HR value because this was generally small compared to the statistical error.

The numbers of sources detected in each camera system and in each energy band are given in Table 2. Of the 424 sources in the catalogue, 132 are detected in both the pn and MOS cameras, which represents 59 per cent of the pn sample but only 38 per cent of the MOS sources. The fact that there are more source detections in the MOS channel than in the pn camera reflects both the lack of pn data for some of the fields and also the longer exposures times typically achieved for the MOS detectors (the set-up time for the pn camera is a significant overhead for these rather short observations). For both the MOS and pn instruments, considerably more sources were detected in the hard energy band than in the soft band. It is surprising that the overlap between the spectral channels, i.e. the number of sources independently detected in both the hard and soft spectral bands, is so small (only $\sim 10-15$ per cent of the sample). This spectral characteristic presumably also explains why the broad-band channel is only marginally more sensitive than its component bands, as demonstrated by the fact that only $\sim 11$ per cent of the sources were detected solely in the broad-band. Ebisawa
Table 2. Summary of source detections in each camera and energy band.

\begin{tabular}{lcccccc}
\hline \multirow{2}{*}{ Camera } & Soft & Hard & Broad & H\&S $^{a}$ & B-only $^{b}$ & Total \\
\hline pn & 90 & 128 & 171 & 22 & 26 & 222 \\
MOS & 135 & 215 & 266 & 43 & 38 & 345 \\
\hline
\end{tabular}

Notes. ${ }^{a}$ Number of sources detected in both the hard and soft bands.

${ }^{b}$ Number of sources detected only in the broad-band.

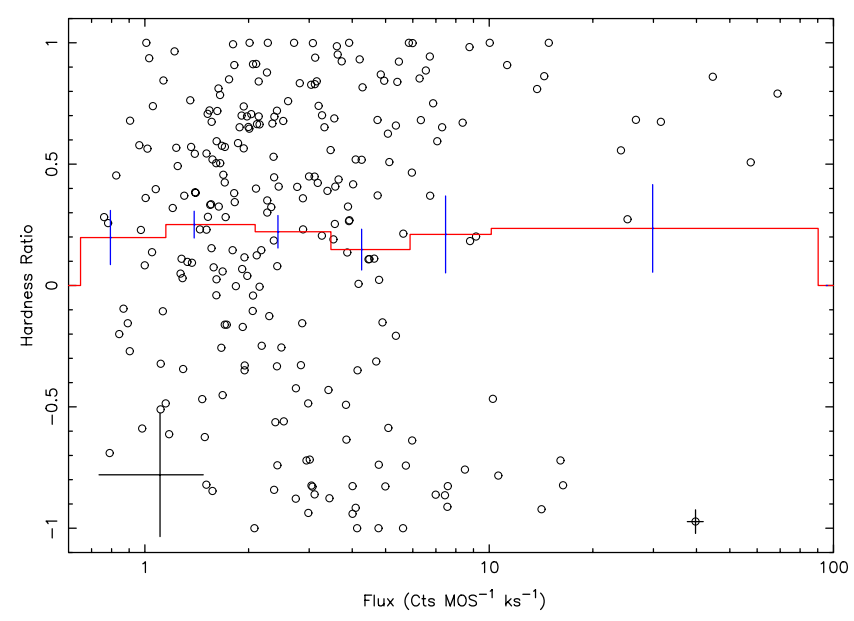

Figure 6. The relationship between spectral hardness and source count rate for sources detected in the MOS cameras. Error bars are shown for representative high and low count-rate points. The histogram charts the variation in the average HR with count rate.

et al. (2001) have noted a similar lack of overlap between the soft and hard source populations detected in deep Chandra observations of the Galactic plane.

\subsection{The spectral properties of the XGPS-I sources}

The range of spectral hardness exhibited by the XGPS-I sources is illustrated in Fig. 6 which shows HR versus MOS count rate for sources detected in the MOS cameras. There is clearly a huge spread encompassing the full range of the HR parameter (i.e. $\mathrm{HR}=-1$ to +1$)$. Given this scatter, it is not surprising that there is little evidence for a variation of the average HR with decreasing count rate (as might be predicted, for example, if fainter sources are on average more distant and as a consequence are more strongly absorbed).

We have investigated the typical spectral form of the XGPS-I source population by considering the integrated spectra of different subsets of sources. In fact, we split the population into three groups depending on the HR parameter as follows: (i) soft sources with $\mathrm{HR}<-0.5$; (ii) mid-range sources with $-0.5 \leqslant \mathrm{HR} \leqslant 0.5$ and (iii) hard sources with $\mathrm{HR}>0.5$. We use the procedure described in Section 3.3 to extract the integrated spectra for the individual fields and then sum over the set of observations to obtain the average spectrum for each source group. To avoid undue bias, an extremely bright source detected in XGPS 9 (XGPS-I J182833-103659 - see Section 4.5), which contains a comparable number of counts to all the other sources put together, was excluded from this process.

The integrated MOS spectra obtained as above were analysed using the XSPEC software package. Following standard practice, the spectra were binned prior to analysis to give a minimum of 20 counts 

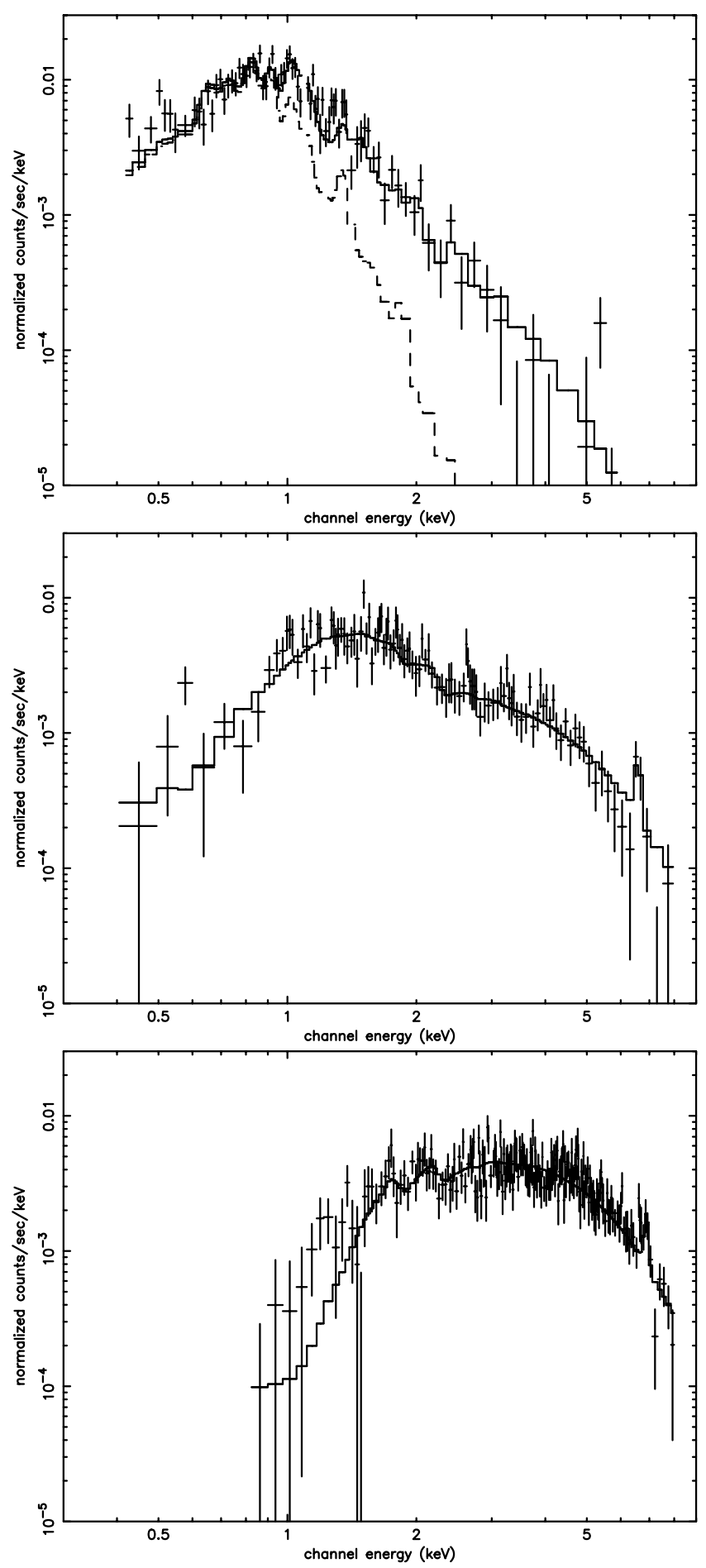

Figure 7. Top panel: the integrated EPIC MOS spectrum of the soft XGPS-I sources with $\mathrm{HR}<-0.5$. Middle panel: the integrated EPIC MOS spectrum of the XGPS-I sources with mid-range hardness ratios i.e. $-0.5 \leqslant \mathrm{HR} \leqslant$ 0.5. Bottom panel: the integrated EPIC MOS spectrum of the hard XGPS-I sources with $\mathrm{HR}>0.5$. In each case the histogram represents the best-fitting spectral model described in the text. In the top panel we also show the contribution of the lower temperature MEKAL component to the measured spectrum.

per spectral channel. From Fig. 7 it is immediately evident that the spectra of the three groups of sources are very different.

Initially we fitted the soft-source spectrum in the $0.4-6.0 \mathrm{keV}$ range with three different models: a power-law, bremsstrahlung and
Table 3. Modelling of the soft-source spectral data.

\begin{tabular}{lccccc}
\hline Model & $N_{\mathrm{H}}^{a}$ & $\Gamma$ & $k T_{1}^{b}$ & $k T^{b}{ }_{2}$ & $\chi^{2}$ (dof) \\
\hline Power-law & $0.65_{-0.05}^{+0.08}$ & $5.8_{-0.03}^{+0.06}$ & - & - & $164(107)$ \\
Brems. & $0.32_{-0.04}^{+0.13}$ & - & $0.38_{-0.02}^{+0.03}$ & - & $173(106)$ \\
1-MEKAL & $\sim 0.60$ & - & $\sim 0.5$ & - & $299(107)$ \\
2-MEKAL & $0.44_{-0.03}^{+0.03}$ & - & $0.25^{c}$ & $1.5^{c}$ & $160(107)$ \\
\hline
\end{tabular}

Notes. ${ }^{a}$ In units of $10^{22} \mathrm{~cm}^{-2} .{ }^{b}$ In keV. ${ }^{c}$ Fixed parameter.

MEKAL thermal plasma model, including absorption in each case. The pure continuum models provided the best fits (albeit with modest reduced $\chi^{2}$ ), with the power-law model requiring a very steep spectral index and the bremsstrahlung model requiring a relatively low temperature (see Table 3). In contrast, a single-temperature solar-abundance MEKAL model provided a poor fit to the spectrum. However, because the pure continuum models are probably not physically realistic characterizations of this soft spectrum, we also investigated a two-component solar-abundance MEKAL model (plus absorption). The result, with the two-temperature parameters fixed at representative values (here we use $k T=0.25$ and $1.5 \mathrm{keV}$, respectively), was a slight improvement in terms of $\chi^{2}$ to those obtained for the power-law and bremsstrahlung models. Table 3 provides details of the fit and Fig. 7 compares the best-fitting twotemperature model with the data.

An initial investigation of both the mid-range and hard-source spectra (over the spectral range $0.4-8 \mathrm{keV}$ ) demonstrated that a simple power-law continuum plus absorption model provided a good description of both data sets with a fairly similar value for the spectral index $(\Gamma \approx 1.6)$ but with the absorption column density for the mid-range sample significantly lower than for the hard-spectrum sources. On this basis, we fitted the two spectra simultaneously with the absorbed power-law model, but with the spectral index as the only tied parameter. The result was a good fit $\left(\chi^{2}=440\right.$ for 421 dof $)$ with $\Gamma=1.60_{-0.13}^{+0.10}$ and $N_{\mathrm{H}}$ values of $0.5_{-0.08}^{+0.08} \times 10^{22}$ and $3.7_{-0.4}^{+0.3} \times$ $10^{22} \mathrm{~cm}^{-2}$ for the mid-range and hard-source spectra, respectively. With $\Gamma$ fixed at 1.7 the respective $N_{\mathrm{H}}$ values became $0.6_{-0.05}^{+0.06} \times 10^{22}$ and $3.9_{-0.3}^{+0.2} \times 10^{22} \mathrm{~cm}^{-2}$. A comparison of the best-fitting models with the data are again shown in Fig. 7.

Both the mid-range and hard source spectra contain a line emission feature in the 6-7 keV band, consistent with $\mathrm{Fe} \mathrm{K}_{\alpha}$ emission. Although the data are of limited quality, we determine the line centroid values to be $6.59 \pm 0.07$ and $6.88 \pm 0.06 \mathrm{keV}$ for the medium and hard sources, respectively; the equivalent widths are measured to be $370 \pm 250$ and $240 \pm 110 \mathrm{eV}$.

The fact that the soft-source spectrum is well fitted by a canonical two-temperature model with a relatively low absorption column is consistent with the bulk of the soft population being relatively nearby active stars. The spectra of the mid-range and hard-source samples are less easy to characterize. Certainly many of the faint hard sources may be active galactic nuclei (AGN; see Section 5.1) but the relatively hard continuum spectrum and iron-line properties also match the spectral properties of cataclysmic variables (CVs) and RS CVns. For example, CVs often exhibit a two-temperature thermal spectrum with $k T \sim 0.5-1 \mathrm{keV}$ and $\sim 5-10 \mathrm{keV}$ (e.g. Baskill, Wheatley \& Osborne 2004). With significant line of sight absorption the latter component dominates and readily mimics the hard powerlaw form inferred above.

\subsection{The XGPS-I source counts}

In order to study the number density of discrete X-ray sources as a function of count rate it is necessary to correct for the variation in 

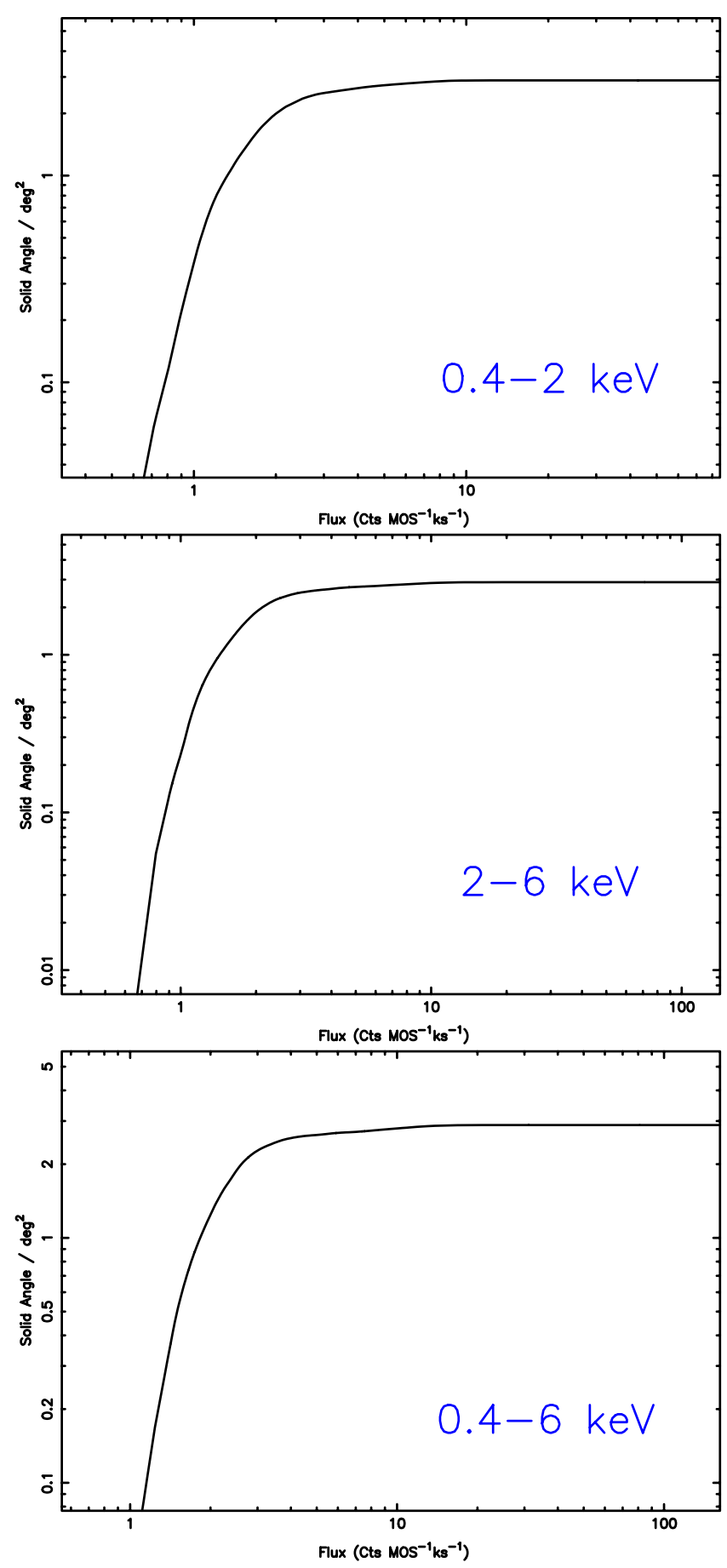

Figure 8. Sensitivity curves for the source count analysis based on detections with the MOS cameras in three energy bands. The total solid angle coverage of the survey (for very bright sources) is $\sim 3 \mathrm{deg}^{2}$.

the source detection sensitivity across the set of XMM fields which comprise the survey. Here we concentrate solely on the sources detected in the MOS cameras.

The first step in the correction process was to calculate a sensitivity map for source detection (in 'on-axis' count-rate units) for each XGPS-I observation based on the exposure map (which accounts for vignetting and other relevant factors such as chip gaps) and the derived MOS background map. The total survey area over which a source of a given count rate was detectable was then readily calculated by summing over the set of sensitivity maps comprising the XGPS-I survey. The derived effective area curves are shown in Fig. 8 for the three energy bands of the survey.

The X-ray source counts are then constructed by summing the contributions of individual sources after correction for the survey sensitivity. For example, consider a source detected at some particular offset angle in one of the XGPS-I observations. Its on-axis count rate is obtained by simply dividing the corrected net counts by the value of the exposure map at the source position. We then use the derived sensitivity curves to determine the solid angle $(\Omega)$ over which a source of that count rate was detectable. This source then contributes $1 / \Omega$ to the source counts at its measured count-rate value. The final source count is obtained by summing the contributions of all the detected sources. ${ }^{7}$

In order to obtain an estimate of the magnitude of the error that should be assigned to the derived source counts at a given flux we have carried out a Monte Carlo simulation of the post-detection process used to construct the source counts (NB bearing in mind that with integral counts the measurements are not independent from point to point). This simulation also demonstrated that the changing gradient of the sensitivity curves at low fluxes introduces a significant bias in the source counts; in effect the Poissonian variation in the measured flux of a source has an asymmetric effect on the value of $\Omega$ that is derived. We correct for this bias by simulating the source counts both with and without such flux errors, noting the differences and adjusting the measured data accordingly. In practice, this procedure resulted in a reduction in the inferred number density of sources at the survey limit by up to 40 per cent.

Fig. 9 shows the corrected integral source counts in the three bands. It is evident that XGPS-I survey detects discrete X-ray sources in the Galactic plane down to surface density of roughly $200 \mathrm{deg}^{-2}$.

By linearly fitting the data in binned, differential form, we determined the slope of the integral counts to be $-1.5 \pm 0.2$ for both the soft and hard sources and $-1.3 \pm 0.2$ for the broad-band sources. (These values represent the slopes of the source counts after excluding, in each case, a handful sources at the bright end of the flux range.)

\subsection{Optical/X-ray source correlations}

Although the X-ray positions typically have statistical errors of $\leqslant 4$ arcsec (see Section 3.2), we have searched for optical counterparts within a nominal 6-arcsec error circle. Specifically we have used optical data from the SUPERCOSMOS digitization of the sky survey plates from the UK Schmidt telescope (UKST). Appendix A identifies the brightest optical source (if any) on the red $(R)$ plate within the error circle of each XGPS-I source and quotes the corresponding optical $R$ magnitude. Cross-references to the optical source in the USNO-A2.0 catalogue and/or the SIMBAD data base are also noted. Of the $424 \mathrm{X}$-ray point sources, 188 have possible optical counterparts identified by this procedure.

The correlation of optical magnitude versus X-ray count rate (here we focus on detections with quality $>0.8$ in the MOS cameras) is a scatter diagram. Similarly a plot of X-ray hardness ratio versus optical $R$ magnitude also shows significant scatter (Fig. 10), although there is hint of X-ray spectral hardening as one goes to optically fainter sources in the range $R=12-18$.

\footnotetext{
${ }^{7}$ Sources with quality $<0.8$, such as those located at a CCD chip edge or strongly affected by bad pixels were excluded.
} 

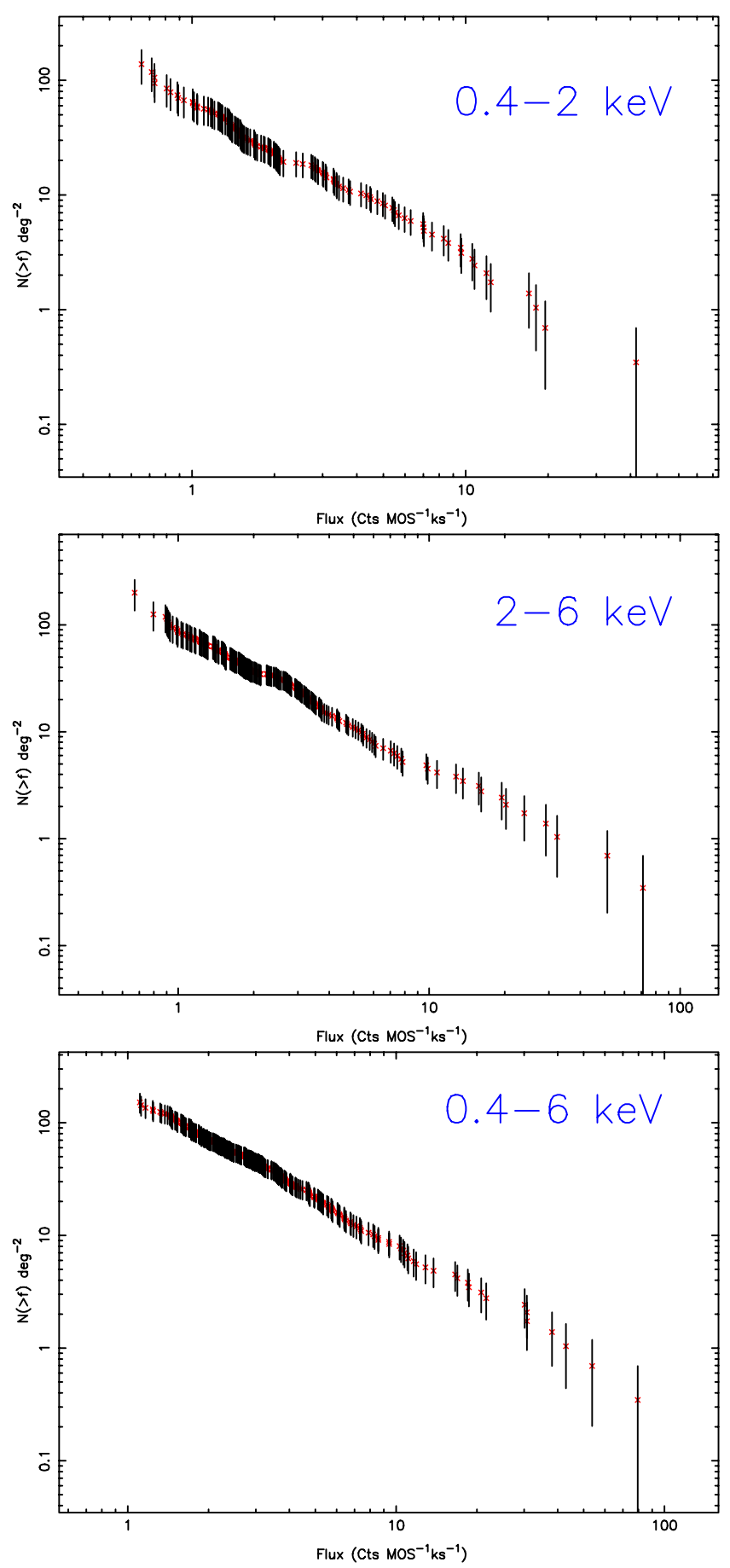

Figure 9. The derived X-ray source counts plotted in three energy bands and corrected at faint fluxes for the bias in the coverage correction induced by flux errors (see text).

We have investigated how the number of optical/X-ray correlations varies with optical magnitude for three subsets of sources divided according to the X-ray hardness ratio (i.e. the soft-, midrange and hard-spectrum samples defined earlier). Fig. 11 shows how the fraction of X-ray sources with an associated optical source rises with increasing $R$. For the hard X-ray source sample, the rate of optical correlation is essentially the same as the chance rate. However, both the soft and mid-range samples have significantly higher

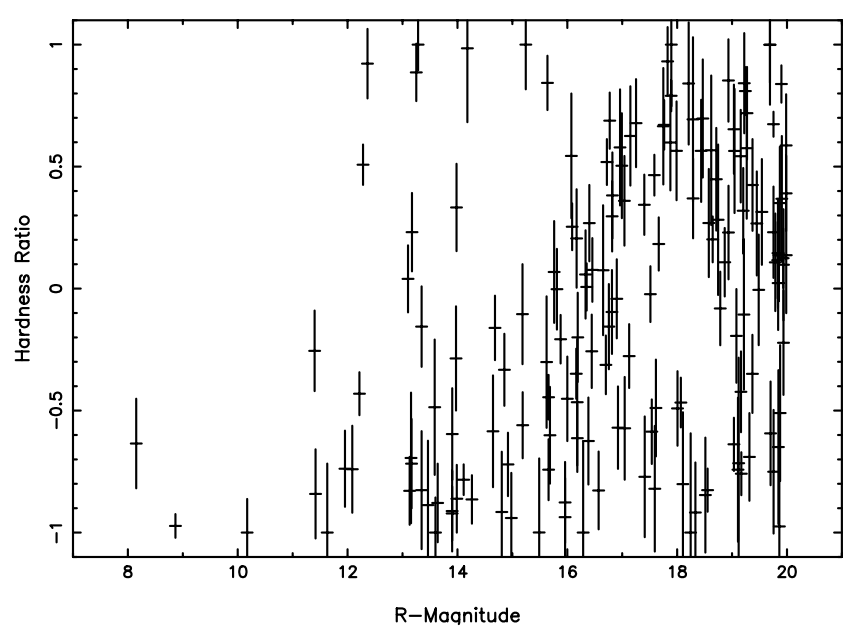

Figure 10. X-ray spectral hardness ratio versus optical $R$-magnitude. This refers to the brightest optical object in the X-ray error circle of each XGPS-I source. The probability that the optical source is the true optical counterpart of the X-ray source decreases both with increasing $R$ and hardness ratio see the discussion in the text.

rates of optical/X-ray associations than expected by chance. For these, we can compare the observed and chance rates to estimate the fraction of genuine optical identifications within the full list of optical associations (see Fig. 11, lower panel).

The X-ray sources with soft spectra $(\mathrm{HR}<-0.5)$ have a particularly high rate of association with bright optical objects. For example, $\sim 45$ per cent of such sources have an optical object brighter than $R=18$ within 6 arcsec of the X-ray position and of these roughly 75 per cent are likely to be the correct counterpart. At $R=20$ the two factors become $\sim 65$ and $\sim 55$ per cent respectively. On the basis of the inferred X-ray/optical ratio and the X-ray spectral characteristics discussed earlier, it is likely that many of these soft X-ray sources are nearby late-type stars with active coronae.

Having identified a subset of the optical/X-ray associations which have a relatively high probability of being the correct identification, we can use the measured optical to X-ray positional offsets to check the astrometry of the X-ray positions, including any component relating to an overall shift (and rotation) of the XMM-Newton reference frame. Fig. 12 shows the radial distribution of the optical/X-ray offsets for the soft sources with associated optical objects brighter than $R=20$. Allowing for a uniform distribution of chance coincidences we find the radius encompassing 68 per cent ( 90 per cent) of the 'real' identifications is $3.3 \operatorname{arcsec}(4.7 \mathrm{arcsec})$ which is comparable to our earlier estimate of the statistical errors associated with the $\mathrm{X}$-ray positions. This analysis demonstrates that any systematic astrometric shift of the XMM-Newton reference frame (for each field) to the true celestial frame must be small (of the order 1-2 arcsec at most), a result that is in accord with other studies (e.g. Barcons et al. 2002; Watson et al. 2003). Of the 22 XGPS fields, 17 have at least one soft source with an optical counterpart brighter than $R=$ 20 within 4.7 arcsec. Conversely, we calculate that an incidence of five fields with zero correlations is not a particularly unlikely event. Unfortunately this does mean that for the latter fields we have no independent check of the XMM-Newton aspect solution (the fields in question are Ridge 4 and XGPS 2, 4, 6 and 14), but we have no reason to believe the astrometric accuracy of any of these fields is anomalous. 

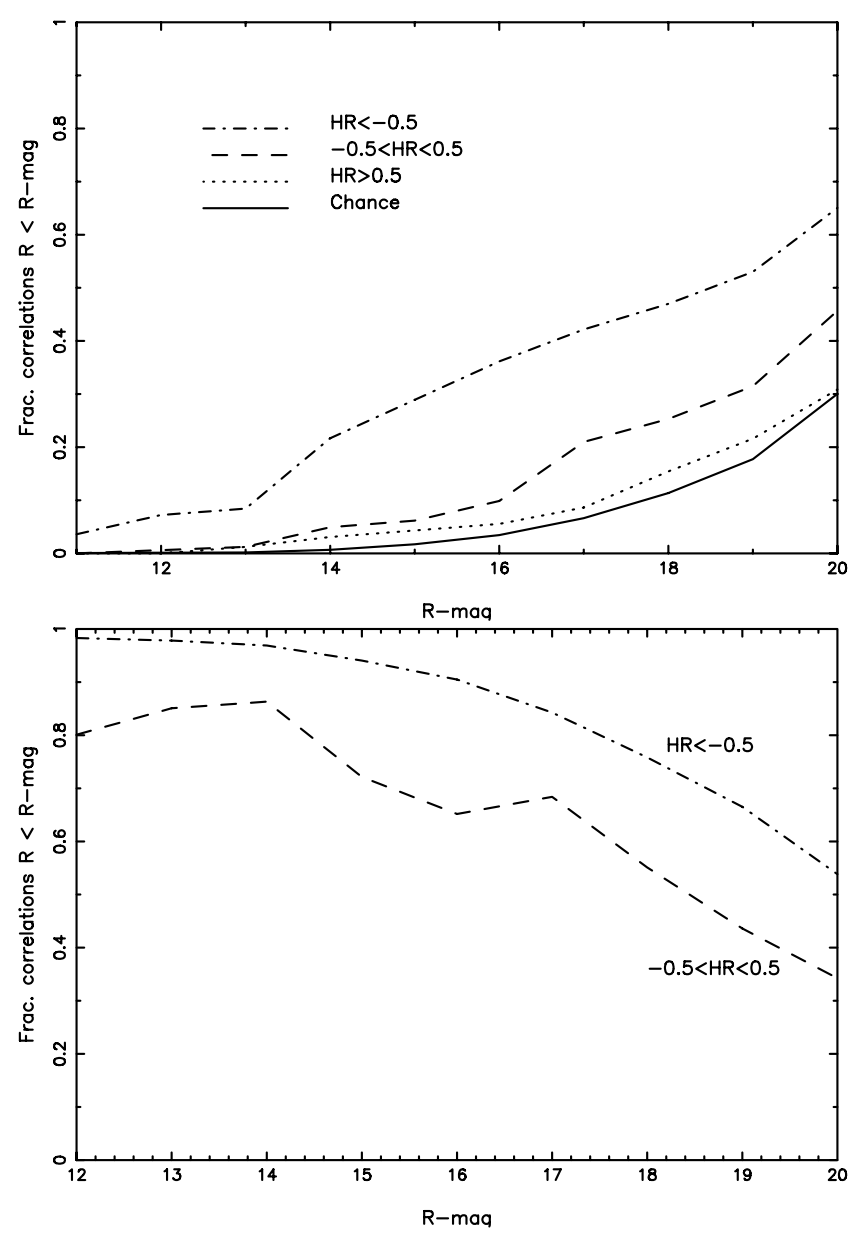

Figure 11. Top panel: the fraction of X-ray sources with an associated optical source plotted versus the limiting $R$ magnitude of the optical sample. The three upper curves correspond to X-ray sources with spectral hardness in three ranges, namely soft sources with $\mathrm{HR}<-0.5$, mid-range sources with $-0.5 \leqslant \mathrm{HR} \leqslant 0.5$ and hard sources with HR $>0.5$. The lower curve shows the chance coincidence rate for finding an optical source in a 6 -arcsec error circle in this region of the sky. Bottom panel: the fraction of the optical/X-ray associations that are likely to represent real identifications. The two curves correspond to the soft (upper) and mid-range (lower) spectral samples.

\subsection{A bright transient source}

Only one source in the XGPS-I catalogue is bright enough to merit individual spectral extraction. The source, designated XGPSI J182833-103659, is located at RA $18^{\mathrm{h}} 28^{\mathrm{m}} 34.0$, Dec. (J2000) $-10^{\circ} 36^{\prime} 59^{\prime \prime}$ (Galactic coordinates $l=20.9, b=0.2$ ). Fitting an absorbed thermal bremsstrahlung model to the measured spectrum yields a temperature of $\sim 7 \mathrm{keV}$ and an absorption column of $\sim 5 \times 10^{22} \mathrm{~cm}^{-2}$ (see Fig. 13). This column density is consistent with either an extragalactic or a distant Galactic origin. In the latter case (assuming a distance of $\sim 15 \mathrm{kpc}$ ) the observed flux is equivalent to an X-ray luminosity of $\sim 10^{35} \mathrm{erg} \mathrm{s}^{-1}$.

Cornelisse et al. (2002) discovered six type I X-ray bursters in BeppoSAX Wide Field Camera (WFC) observations, one of which is positionally coincident with this bright XGPS-I source, although the WFC 99 per cent confidence error circle of 2.8 arcmin is relatively large. The peak flux measured by BeppoSAX for this source (during a burst) was $(1.1 \pm 0.4) \times 10^{-8} \mathrm{erg} \mathrm{s}^{-1} \mathrm{~cm}^{-2}(2-10 \mathrm{keV})$ with a burst duration of $\sim 30 \mathrm{~s}$. In the same energy band we measure $\sim 7.5 \times 10^{-12} \mathrm{erg} \mathrm{s}^{-1} \mathrm{~cm}^{-2}$ (after correcting for absorption). This

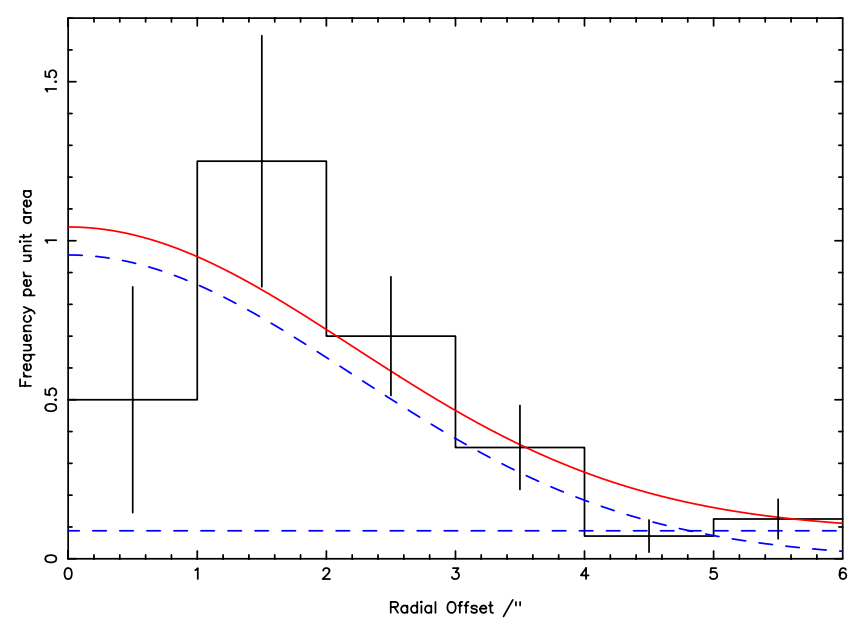

Figure 12. The distribution of the optical/X-ray offsets measured for the soft source sample in radial bins of 1 -arcsec width. The error bars correspond to $\pm 1 \sigma$. The solid-line represents the sum of the uniform distribution of the chance coincidences and the assumed Gaussian distribution of the real identifications. The latter has $\sigma=2.2$ arcsec corresponding to a 68 per cent (90 per cent) probability error circle radius of $3.3 \operatorname{arcsec}(4.7 \operatorname{arcsec})$.

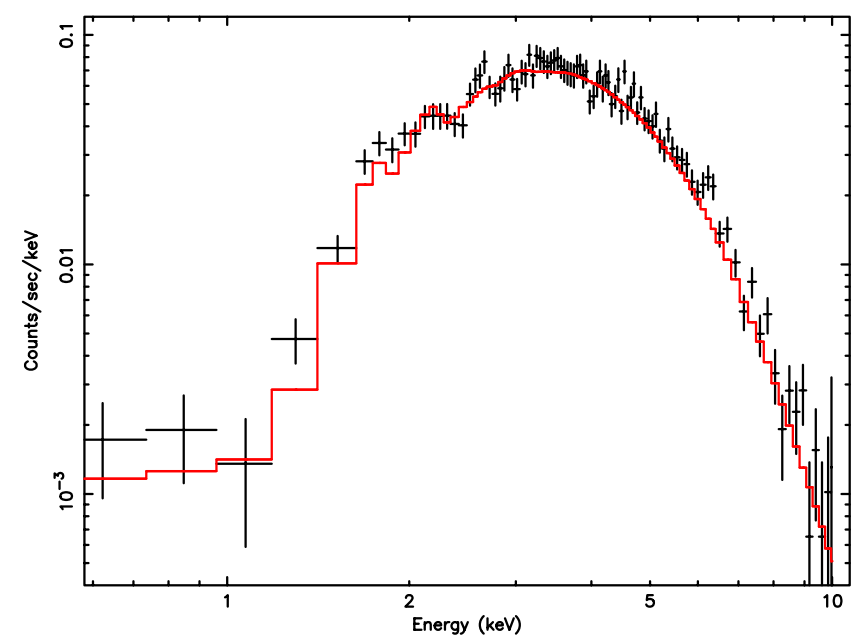

Figure 13. The measured count-rate spectrum of a bright transient source. The histogram corresponds to the best-fitting model detailed in the text.

is more than three orders of magnitude fainter than the burst peak, but may be only a factor $\sim 10$ fainter than the high-state persistent flux of this source for which Cornelisse et al. (2002) quote only an upper limit of $<1.9 \times 10^{-10} \mathrm{erg} \mathrm{s}^{-1} \mathrm{~cm}^{-2}$. XGPS-I J182833-103659 shows no variation in its light curve over the short XMM-Newton observation, indicating that we are detecting persistent emission. The source position was in fact covered by two XGPS-I observations, XGPS 9 and Ridge 5, but the source was detected only in the former. This places an upper flux limit on the low state of the source of approximately $2 \times 10^{-14} \mathrm{erg} \mathrm{s}^{-1} \mathrm{~cm}^{-2}$ in the $2-10 \mathrm{keV}$ band. This source thus clearly shows significant variability: by a factor $\sim 300$ between XMM-Newton observations and quite possibly by a much larger factor overall, making it very likely to be a previously unrecognised X-ray transient source. X-ray bursts are of course commonly associated with X-ray transient systems. The observed low state is also consistent with the absence of the source 
Table 4. Correlation of XGPS-I sources with the ASCA catalogue.

\begin{tabular}{lc}
\hline XMM ID & ASCA name \\
\hline XGPS-I J182534-121454 & AX J182538-1214 \\
XGPS-I J182846-111711 & AX J182846-1116 \\
XGPS-I J182525-114525 & AX J182530-1144 \\
XGPS-I J183038-100249 & AX J183039-1002 \\
XGPS-I J183117-100921 & AX J183116-1008 \\
XGPS-I J183209-093906 & AX J183206-0938/AX J183206-0940 \\
- & AX J183114-0943 \\
- & AX J182651-1206 \\
\hline
\end{tabular}

from the catalogue derived from the ASCA Galactic Plane Survey (Sugizaki et al. 2001).

\subsection{Comparison with $A S C A$}

The region surveyed by XGPS-I is entirely covered by the ASCA Galactic Plane Survey (Sugizaki et al. 2001) which resolved 163 sources in the Galactic plane within a longitude span of $90^{\circ}$ centred on $l=0^{\circ}$. Of these 163 sources nine fall within the nominal region covered by the XGPS-I observations. We have detected seven of these sources as summarized in Table 4. Note that, two of the ASCA sources are linked to the same XGPS-I source as a consequence of the relatively poor spatial resolution and large positional errors of the former.

\section{DISCUSSION}

\subsection{Source populations and the $\log N-\log S$ relation}

The source number versus flux $(\log N-\log S)$ relation can provide important information on the spatial distribution and the luminosity functions of the various Galactic source populations. Here we combine our present measurements with those from other missions to examine how various categories of source may contribute to the observed hard band source counts. An investigation of the soft band $\log N-\log S$, including a comparison with earlier ROSAT measurements, will be the subject of a future paper.

In order to relate the XMM-Newton measurements to observations from other satellites it is necessary to convert the measured source counts from count-rate to flux units. Table 5 lists the conversion factors from MOS count rate in the $2-6 \mathrm{keV}$ band to the corresponding flux (in erg s $\mathrm{cm}^{-1} \mathrm{~cm}^{-2}$ ) in the $2-10 \mathrm{keV}$ band for a variety of spectral forms calculated using PIMMS (Mukai 1993).

In the present analysis we adopt a factor $2.6 \times 10^{-14} \mathrm{erg}$ $\mathrm{s}^{-1} \mathrm{~cm}^{-2} / \mathrm{MOS}$ count $\mathrm{ks}^{-1}$ corresponding to a power-law source spectrum with spectral index $\Gamma=1.7$ absorbed by a column density $N_{\mathrm{H}}=1 \times 10^{22} \mathrm{~cm}^{-2}$. This is clearly a compromise given the range of spectral form established earlier (see Section 4.2); we estimate

Table 5. Factors ${ }^{a}$ to convert from the MOS count rate in the $2-6 \mathrm{keV}$ band to the observed flux in the $2-10 \mathrm{keV}$ band for different power-law spectra with a range of interstellar absorptions.

\begin{tabular}{llllll}
\hline$\Gamma \backslash N_{\mathrm{H}}{ }^{b}$ & 0.0 & 1.0 & 3.0 & 5.0 & 7.0 \\
\hline 1.4 & 2.7 & 3.0 & 3.4 & 3.9 & 4.4 \\
1.7 & 2.4 & 2.6 & 3.0 & 3.4 & 3.9 \\
2.0 & 2.1 & 2.3 & 2.7 & 3.1 & 3.4 \\
\hline
\end{tabular}

Notes. ${ }^{a}$ In units of $10^{-14} \mathrm{erg} \mathrm{s}^{-1} \mathrm{~cm}^{-2} /\left(\operatorname{MOS}\right.$ count $\left.\mathrm{ks}^{-1}\right) .{ }^{b}$ In units of $10^{22} \mathrm{~cm}^{-2}$.

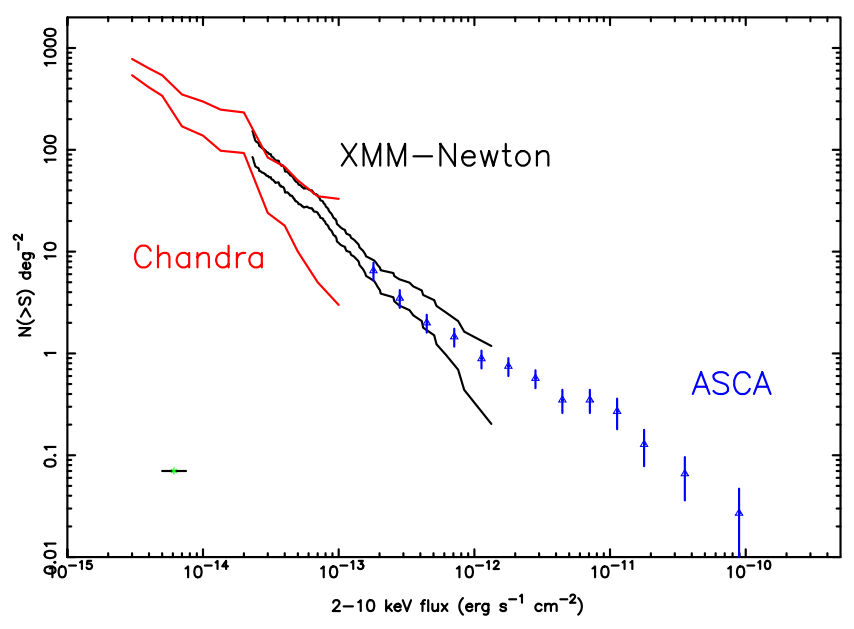

Figure 14. The $2-10 \mathrm{keV} \log N-\log S$ relation measured in the Galactic plane based on ASCA, XMM-Newton and Chandra observations (see the text for references). In the case of both the XMM-Newton and Chandra measurements we show the upper and lower bounds of the derived source counts rather than individual data points. The magnitude of the relative flux scaling uncertainty applicable to the three data sets is indicated by the horizontal error bar.

that the effective uncertainty in the flux scaling may be as large as \pm 30 per cent.

Fig. 14 shows the measured $\log N-\log S$ relation in the $2-10 \mathrm{keV}$ band based on the XGPS-I measurements. Here we have clipped one high flux point with large error bars and two low-flux points requiring very large coverage correction, from the equivalent representation in Fig. 9. For comparison the source counts derived from the extensive survey of the Galactic plane between $l= \pm 45^{\circ}$ carried out by ASCA (Sugizaki et al. 2001) and from recent deep Chandra observations in the Galactic plane at $l=28^{\circ}$ (Ebisawa et al. 2001) are also shown.

As can be seen from Fig. 14, the flux range probed by the XGPS-I measurements is intermediate between that sampled by the ASCA and Chandra programmes. The agreement between the XGPS-I and $A S C A$ surveys is rather good given the very different coverage of the Galactic plane inherent in the two programmes. The agreement between the XGPS-I and Chandra source counts is also good bearing in mind the different pointing directions and the fact that at $\sim 3 \times$ $10^{-14} \mathrm{erg} \mathrm{s}^{-1} \mathrm{~cm}^{-2}$ there are only $\sim 6$ sources in the latter survey. Based on this compilation, the $\log N-\log S$ relation appears to first flatten, then steepen, then flatten again as one moves from bright sources at $\sim 10^{-10} \mathrm{erg} \mathrm{s}^{-1} \mathrm{~cm}^{-2}$ to faint sources at a limiting flux of $\sim 3 \times 10^{-15} \mathrm{erg} \mathrm{s}^{-1} \mathrm{~cm}^{-2}$ in the $2-10 \mathrm{keV}$ band.

The first step in modelling the measured composite $\log N-\log S$ relation in terms of various underlying source populations is to quantify the contribution of extragalactic sources. It has in fact been recently demonstrated that even in heavily obscured regions of the Galactic plane the X-ray source counts measured at faint fluxes in the hard band are dominated by this component (Ebisawa et al. 2001). The extragalactic $\log N-\log S$ relation in the $2-10 \mathrm{keV}$ band has been determined over a wide range of X-ray flux from HEAO-1 A2 observations at the bright end (Piccinotti et al. 1982) through to recent ultradeep Chandra observations which probe below $\sim 10^{-15} \mathrm{erg} \mathrm{s}^{-1} \mathrm{~cm}^{-2}$ (Rosati et al. 2002; Cowie et al. 2002; Alexander et al. 2003; Moretti et al. 2003; Harrison et al. 2003). Here we use the empirical form for the extragalactic $2-10 \mathrm{keV}$ $\log N-\log S$ specified by Campana et al. (2001) based on a comparison of deep Chandra observations with ASCA, BeppoSAX and 
other data sets. The integral form of the extragalactic source counts flatten from a power-law slope of -1.67 at intermediate fluxes to a value of -0.58 at faint fluxes with the break occurring near $\sim 2 \times$ $10^{-14} \mathrm{erg} \mathrm{s}^{-1} \mathrm{~cm}^{-2}$.

A very important factor in modelling the extragalactic contribution to the Galactic plane $\log N-\log S$ is the signal loss due to absorption in the line-of-sight column density through Galaxy. Our spectra analysis of the sources with the hardest spectra (see Section 4.2) sets a lower limit of $N_{\mathrm{H}}=3.9 \times 10^{22} \mathrm{~cm}^{-2}$ for an assumed power-law source spectrum with $\Gamma=1.7$, whereas the bright transient source discussed earlier (Section 4.5) required $N_{\mathrm{H}}=5.0 \times$ $10^{22} \mathrm{~cm}^{-2}$. By way of comparison, Ebisawa et al. (2001) argue that $N_{\mathrm{H}}=4-6 \times 10^{22} \mathrm{~cm}^{-2}$ at $l=28^{\circ}$ when one accounts for both neutral and molecular hydrogen along the line of sight. Based on the H I measurements of Dickey \& Lockman (1990) and molecular hydrogen measurements of Dame, Hartmann \& Thaddeus (2001), one might infer a similar value for the XGPS-I region. On the other hand, Nevalainen et al. (2001) measure a foreground Galactic column density of $7.9 \pm 0.5 \times 10^{22} \mathrm{~cm}^{-2}$ for a cluster of galaxies at $(l, b)=(21.3,-0.7)$. Here we adopted a hard band transmission factor of 0.68 corresponding to a line-of-sight column density of $5 \times 10^{22} \mathrm{~cm}^{-2}$ (for a power-law $\Gamma=1.7$ source spectrum) which, in broad terms, aligns the extragalactic prediction with the observed Chandra source counts at faint fluxes (see Fig. 15). Clearly variation in the Galactic $N_{\mathrm{H}}$ from field to field in the Galactic plane will introduce a significant variance in the extragalactic contamination of the $\log N-\log S$ relation; of necessity here we present only an approximate description of a very complicated situation.

We have investigated the possible contribution of various Galactic source populations to the measured composite $\log N-\log S$ relation through the use of relatively simple prescriptions for the source luminosity function, the source distribution in the Galaxy and the effects of absorption. In brief, the predicted source counts are calculated by a numerical integration along a line of sight at $(l, b)$ $=(20,0)$. We assume the maximum diameter of the Galaxy is 20 $\mathrm{kpc}$ and the Galactocentric radius of the Sun is $8.5 \mathrm{kpc}$. We model the absorption in the plane in terms of a local hydrogen density of $0.55 \mathrm{~cm}^{-3}$. We assume the source and particle densities decline exponentially with respect to Galactocentric radius $\left(R_{\mathrm{G}}\right)$ and height above the plane $(z)$ (the assumed scale factors were $8500 \mathrm{kpc}$ and $200 \mathrm{pc}$ in $R_{\mathrm{G}}$ and $z$ for the sources and $8500 \mathrm{kpc}$ and $100 \mathrm{pc}$ for the particle density).

We first consider relatively luminous Galactic X-ray binary sources containing either a neutron star or (in a few cases) a stellar mass black-hole. Low-mass X-ray binaries (LMXBs) are found preferentially in the Galactic Bulge and Galactic Centre regions whereas high-mass X-ray binaries (HMXRB) tend to avoid the inner 3-4 kpc of the Galaxy but are widely distributed in the Galactic disc (Grimm, Gilfanov \& Sunyaev 2002). The composite $\log N-\log S$ measured at $l=20^{\circ}$ might therefore include contributions from both populations. In order to model the combined LMXB/HMXRB contribution we assume a power-law form for luminosity function with a slope of -1.3 in the differential form (cf. Grimm et al. 2002). In practice, a luminosity function restricted to the range $10^{34}-10^{36}$ $\mathrm{erg} \mathrm{s}^{-1}$ proved sufficient to account for the observed form of the $\log N-\log S$ relation at the bright source end (see Fig. 15). The normalization of the binary luminosity function needed to match the $\log N-\log S$ relation translates via the source distribution model to a Galactic population of $\sim 200$ such X-ray binaries with an integrated Galactic X-ray luminosity of $\sim 1.6 \times 10^{37} \mathrm{erg} \mathrm{s}^{-1}$.

With the bright and faint ends of the measured $\log N-\log S$ relation represented respectively by Galactic X-ray binaries and the breakthrough of extragalactic sources, an excess number of sources (relatively to the prediction) is most apparent in the flux range $10^{-13}$ to $10^{-12} \mathrm{erg} \mathrm{s}^{-1} \mathrm{~cm}^{-2}$. The requirement on any source population invoked to fill this gap is that its source count must be relatively steep at the top end of this range but should gradually turn over below $10^{-13} \mathrm{erg} \mathrm{s}^{-1} \mathrm{~cm}^{-2}$, so as not to overpredict the total source density in the flux range sampled by the Chandra observations.

For illustrative purposes we consider a source population with an X-ray luminosity function described by a log-normal function centred on $L_{\mathrm{X}}=10^{31} \mathrm{erg} \mathrm{s}^{-1}$ with $\sigma=1.0$ and a local spatial density of $\sim 10^{-6} \mathrm{pc}^{-3}$. A source with $L_{\mathrm{X}}=10^{31} \mathrm{erg} \mathrm{s}^{-1}$, at a distance of $1 \mathrm{kpc}$ has an X-ray flux of $10^{-13} \mathrm{erg} \mathrm{s}^{-1} \mathrm{~cm}^{-2}$. At larger distances (and hence lower fluxes), the effects of increasing absorption will serve to flatten the counts of such sources. In addition by $\sim 10^{-14} \mathrm{erg}$ $\mathrm{s}^{-1} \mathrm{~cm}^{-2}$ the most luminous sources in the population are detectable out to the edge of the Galaxy with the result that the overall $\log N-$ $\log S$ relation flattens further. Fig. 15 shows the predicted source count relation for the low-luminosity source population considered above. In combination the two Galactic source populations plus the extragalactic component provide an excellent match to the observed composite $\log N-\log S$ curve.

What class of X-ray source might comprise the low-luminosity population considered above? The most likely candidate population is cataclysmic variables (CVs), close binary systems in which a white dwarf accretes material from a Roche-lobe filling late-type companion. CVs are often relatively bright hard X-ray sources with $L_{\mathrm{X}}=10^{30-32} \mathrm{erg} \mathrm{s}^{-1}$ (e.g. Verbunt et al. 1997). In an earlier analysis, Watson (1999) suggested that X-ray faint CVs might show up in large numbers in deep Galactic surveys carried out in the hard X-ray band if their typical X-ray luminosity is $L_{\mathrm{X}}=10^{31} \mathrm{erg}$ $\mathrm{s}^{-1}(2-10 \mathrm{keV})$ and space density is $\sim 10^{-5} \mathrm{pc}^{-3}$. The latter value is compatible with the $\mathrm{CV}$ space density derived empirically by Patterson (1984) and is not out of line with at least some theoretical estimates (e.g. Kolb 1993). In this context, the space density assumed in our modelling of the low-luminosity population becomes a rather conservative requirement, particularly because other categories of source, such as RS CVn binaries (e.g. Makarov 2003, and references therein) and Be-star X-ray binaries in quiescence (e.g. Terman, Taam \& Savage 1998), might also contribute to the low-luminosity population. The overall requirement is for a Galactic population of $1.2 \times 10^{5}$ objects which produce a Galactic $\mathrm{X}$-ray luminosity of $1.3 \times 10^{37} \mathrm{erg} \mathrm{s}^{-1}$ in the $2-10 \mathrm{keV}$ band, comparable to the integrated X-ray luminosity inferred earlier for the X-ray binary population.

\subsection{Contribution of discrete sources to the GRXE}

Using all XGPS-I observations except the two most contaminated by flaring (XGPS 14, XGPS 15) and the one containing the bright transient source (XGPS 9), we have measured the total full-field MOS count rate (including the resolved sources) in the hard $X M M-$ Newton band (2-6 keV). After correcting for the underlying instrumental background (e.g. see Willingale et al. 2003) and for mirror vignetting of the sky background signal, we obtain $3.7 \pm 0.1$ MOS count $\mathrm{s}^{-1} \mathrm{deg}^{-2}$. This signal is substantially larger than that measured at high Galactic latitude in the MOS cameras consistent with the presence of the GRXE in the field of view for all the XGPS-I pointings.

Applying the same count-rate to flux conversion factor as used in Section 5.1, the total surface brightness of the GRXE corresponds to $9.6 \times 10^{-11} \mathrm{erg} \mathrm{s}^{-1} \mathrm{~cm}^{-2} \mathrm{deg}^{-2}$ in the $2-10 \mathrm{keV}$ band. Earlier estimates put the value variously at $11 \times 10^{-11} \mathrm{erg} \mathrm{s}^{-1} \mathrm{~cm}^{-2} \mathrm{deg}^{-2}$ 


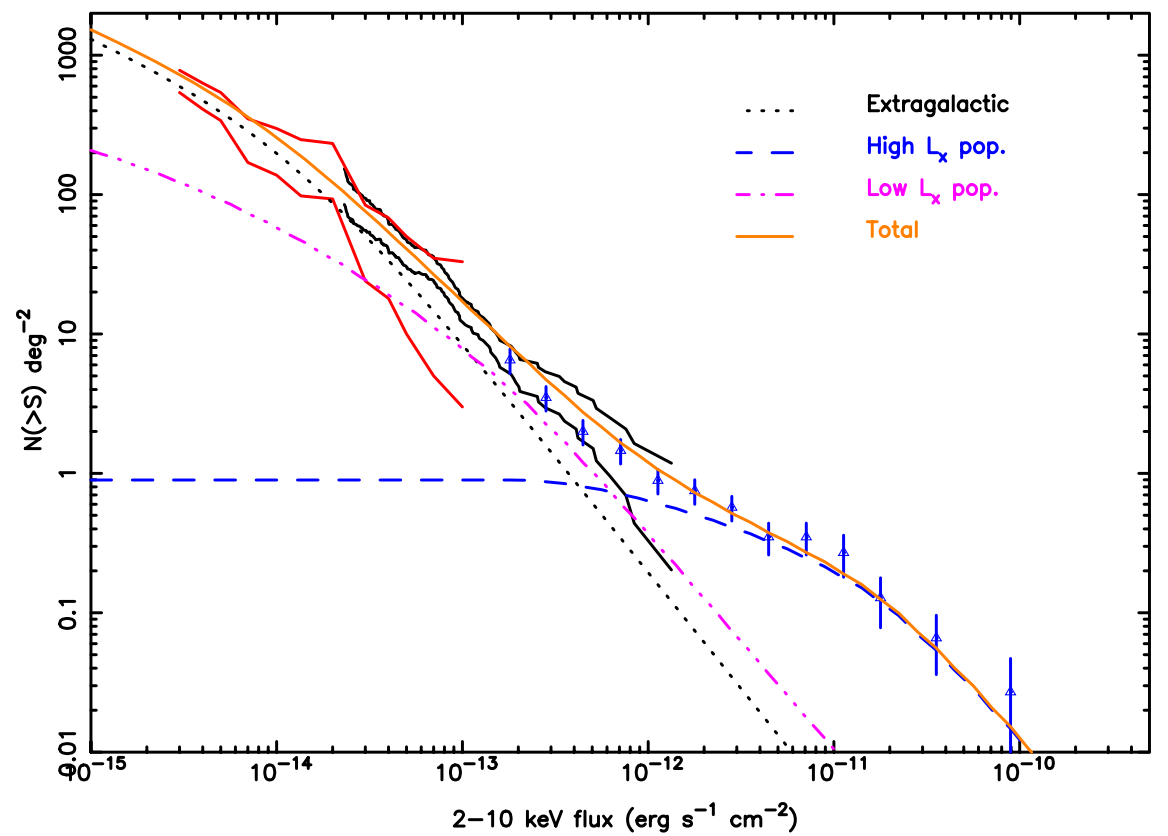

Figure 15. The measured $2-10 \mathrm{keV} \log N-\log S$ relation compared with with the predicted contributions of various X-ray source populations (see the text).

(Chandra; Ebisawa et al. 2001), $5.2 \times 10^{-11} \mathrm{erg} \mathrm{s}^{-1} \mathrm{~cm}^{-2} \mathrm{deg}^{-2}$ (ASCA; Sugizaki et al. 2001), and $2.5 \times 10^{-11} \mathrm{erg} \mathrm{s}^{-1} \mathrm{~cm}^{-2} \mathrm{deg}^{-2}$ (RXTE; Valinia \& Marshall 1998), depending on the region of sky surveyed.

By integrating the observed hard band X-ray source counts we find that the resolved sources with count rates in the range 0.7 to 70 MOS count ks ${ }^{-1}$ (2-6 keV) (on-axis) contribute 0.34 count $\mathrm{s}^{-1} \mathrm{deg}^{-2}$, corresponding to 9 per cent of the observed surface brightness. In addition, we estimate that the contribution of the residual extragalactic background after transmission through Galactic $N_{\mathrm{H}}$ of $5 \times 10^{22} \mathrm{~cm}^{-2}$ amounts to a further 10 per cent of the measured surface brightness. Because the extrapolation of the $\log N-\log S$ curves for the high-luminosity and low-luminosity Galactic source populations below the XGPS-I detection threshold adds little to the integrated signal, the implication is that $\sim 80$ per cent of the measured GRXE surface brightness remains unaccounted for.

The deep Chandra observations show that extragalactic sources dominate down to fluxes of $\sim 3 \times 10^{-15} \mathrm{erg} \mathrm{s}^{-1} \mathrm{~cm}^{-2}(2-10 \mathrm{keV})$ (Ebisawa et al. 2001). A new Galactic population, contributing significantly to the GRXE, might emerge at fainter fluxes but the requirement (deduced by scaling the properties of the low-luminosity population considered earlier) of, say, $L_{\mathrm{X}}=10^{28} \mathrm{erg} \mathrm{s}^{-1}$ combined with a space density of $10^{-2} \mathrm{pc}^{-3}$, does not fit any known population of sources. It would appear therefore that the bulk of the GRXE is truly diffuse in origin although the origin is still uncertain. Possible mechanisms include the interaction of low-energy cosmic-ray electrons or ions with interstellar matter (Valinia et al. 2000; Tanaka 2002), in situ electron acceleration (Dogiel et al. 2002; Masai et al. 2002) and magnetic reconnection (Tanuma et al. 2001). This issue will be addressed in a later paper on diffuse emission from the XGPS survey region.

\section{CONCLUSION}

The XGPS-I survey, which covers approximately $3 \mathrm{deg}^{2}$ of the Galactic plane near $l=20^{\circ}$, has resulted in a catalogue containing over 400 discrete X-ray sources. The measured X-ray source counts trace the source population down to a limiting flux of $\sim 2 \times 10^{-14}$ $\mathrm{erg} \mathrm{s}^{-1} \mathrm{~cm}^{-2}$ in the $2-10 \mathrm{keV}$ band at which point the source density is between 100-200 sources per square degree. Consistent with an earlier Chandra study, the source counts at this flux are predominately due to extragalactic sources, despite the fact that the fluxes of extragalactic objects are significantly suppressed by absorption in the Galactic plane. However, the conclusion of the present work is that at fluxes above $10^{-13} \mathrm{erg} \mathrm{s}^{-1} \mathrm{~cm}^{-2}(2-10 \mathrm{keV})$ Galactic source populations do come to the fore.

The Galactic source population observed between $10^{-13}$ and $10^{-12} \mathrm{erg} \mathrm{s}^{-1} \mathrm{~cm}^{-2}$ could comprise largely CVs and RS CVn systems with X-ray luminosities in the range $10^{30-32} \mathrm{erg} \mathrm{s}^{-1}$ but the details remain uncertain on the basis of the $\mathrm{X}$-ray information alone. Extensive programmes to identify and characterize optical/infrared counterparts are required, although this will be taxing given the high obscuration and high object density in the Galactic plane.

The present work demonstrates that the strategy of the XGPS programme, namely the use of shallow observations to give relatively wide angle coverage is close to optimum in terms of maximizing the number of Galactic source detections.

\section{ACKNOWLEDGMENTS}

ADPH acknowledges support from PPARC in the form of a research studentship and DJH acknowledges support from NASA grant NAG5-9870. We are also very happy to acknowledge the underpinning contributions made by the ESA Science Operations (SOC) team and the XMM-Newton EPIC and SSC consortia to the research programme reported here.

\section{REFERENCES}

Alexander D. M. et al., 2003, AJ, 126, 539

Barcons X. et al., 2002, A\&A, 382, 522

Baskill D. S., Wheatley P. J., Osborne J. P., 2004, MNRAS, submitted

Campana S., Moretti A., Lazzati D., Tagliaferri G., 2001, ApJ, 560, L19 
Cornelisse R. et al., 2002, A\&A, 392, 885

Cowie L. L., Garmire G. P., Bautz M. W., Barger A. J., Brandt W. N., Hornschemeier A. E., 2002, ApJ, 566, L5

Dame T. M., Hartmann D., Thaddeus P., 2001, ApJ, 547, 792

Dickey J. M., Lockman F. J., 1990, ARA\&A, 28, 215

Dogiel V. A., Inoue H., Masai K., Schönfelder V., Strong A. W., 2002, ApJ, 581,1061

Ebisawa K., Maeda Y., Kaneda H., Yamauchi S., 2001, Sci, 293, 1633

Grimm H.-J., Gilfanov M., Sunyaev R., 2002, A\&A, 391, 923

Harrison F. A., Eckart M. E., Mao P. H., Helfand D. J., Stern D., 2003, ApJ, 596,944

Hertz P., Grindlay J. E., 1984, ApJ, 278, 137

Kaneda H., Makishima K., Yamauchi S., Koyama K., Matsuzaki K., Yamasaki N. Y., 1997, ApJ, 491, 638

Kolb U., 1993, A\&A, 271, 149

Koyama K., Makishima K., Tanaka Y., Tsunemi H., 1986, PASJ, 38, 121

Makarov V. V., 2003, AJ, 126, 1996

Masai K., Dogiel V. A., Inoue H., Schönfelder V., Strong A. W., 2002, ApJ, 581,1071

Moretti A., Campana S., Lazzati D., Tagliaferri G., 2003, ApJ, 588, 696

Motch C. et al., 1991, A\&A, 246, L24

Motch C., Guillout P., Haberl F., Pakull M., Pietsch W., Reinsch K., 1997, A\&A, 318, 111

Mukai K., 1993, Legacy, 3, 21

Nevalainen J., Lumb D., dos Santos S., Siddiqui H., Stewart G., Parmar A. N., 2001, A\&A, 374, 66

Patterson J., 1984, ApJS, 54, 443

Piccinotti G., Mushotzky R. F., Boldt E. A., Holt S. S., Marshall F. E., Serlemitsos P. J., Shafer R. A., 1982, ApJ, 253, 485
Rosati P. et al., 2002, ApJ, 566, 667

Strüder L. et al., 2001, A\&A, 365, L18

Sugizaki M., Mitsuda K., Kaneda H., Matsuzaki K., Yamauchi S., Koyama K., 2001, ApJ, 134, 77

Tanaka Y., 2002, A\&A, 382, 1052

Tanuma S., Yokoyama T., Kudoh T., Shibata K., 2001, ApJ, 551, 312

Terman J. L., Taam R. E., Savage C. O., 1998, MNRAS, 293, 113

Turner M. J. L. et al., 2001, A\&A, 365, L18

Valinia A., Marshall F., 1998, ApJ, 505, 134

Valinia A., Tatischeff V., Arnaud K., Ebisawa K., Ramaty R., 2000, ApJ, 543,733

Verbunt F., Bunk W. H., Ritter H., Pfeffermann E., 1997, A\&A, 327, 602

Warwick R. S., Turner M. J. L., Watson M. G., Willingale R., 1985, Nat, 317, 218

Watson M. G., 1999, in Hellier C., Mukai K., ASP Conf. Ser. Vol. 157, Annapolis Workshop on Magnetic Cataclysmic Variables. Astron. Soc. Pac., San Francisco, p. 291

Watson M. G. et al., Astron. Nachr., 324, 89

Willingale R., Hands A. D. P., Warwick R. W., Snowden S. L., Burrow D. N., 2003, MNRAS, 343, 995

Worrall D., Marshall F., Boldt E., Swank J., 1982, ApJ, 255, 111

Yamauchi S., Koyama K., 1993, ApJ, 404, 620

\section{APPENDIX A: THE XGPS-I SOURCE CATALOGUE}

This paper has been typeset from a $\mathrm{T}_{\mathrm{E}} \mathrm{X} / \mathrm{L} \mathrm{A} \mathrm{E} \mathrm{X}$ file prepared by the author. 


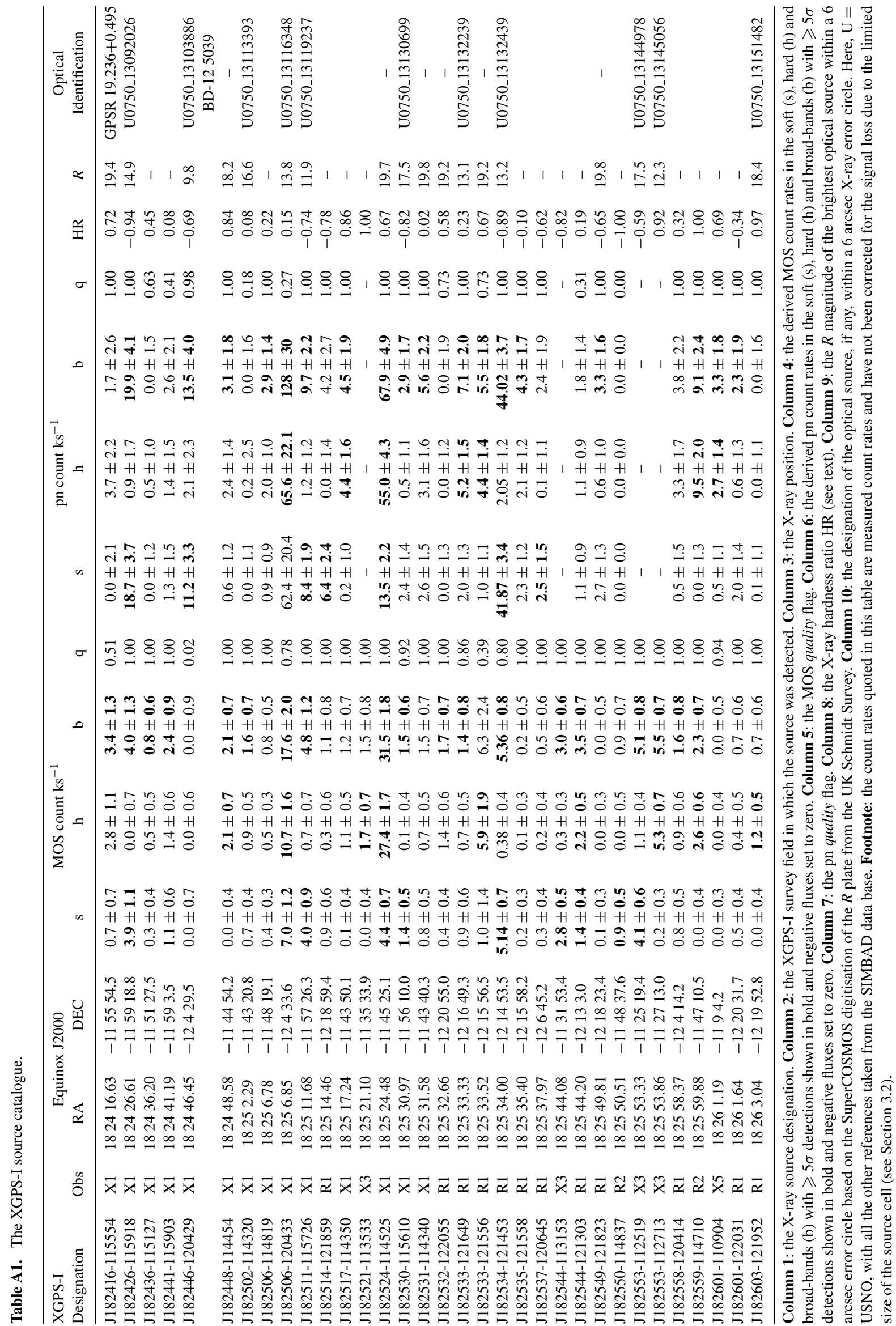




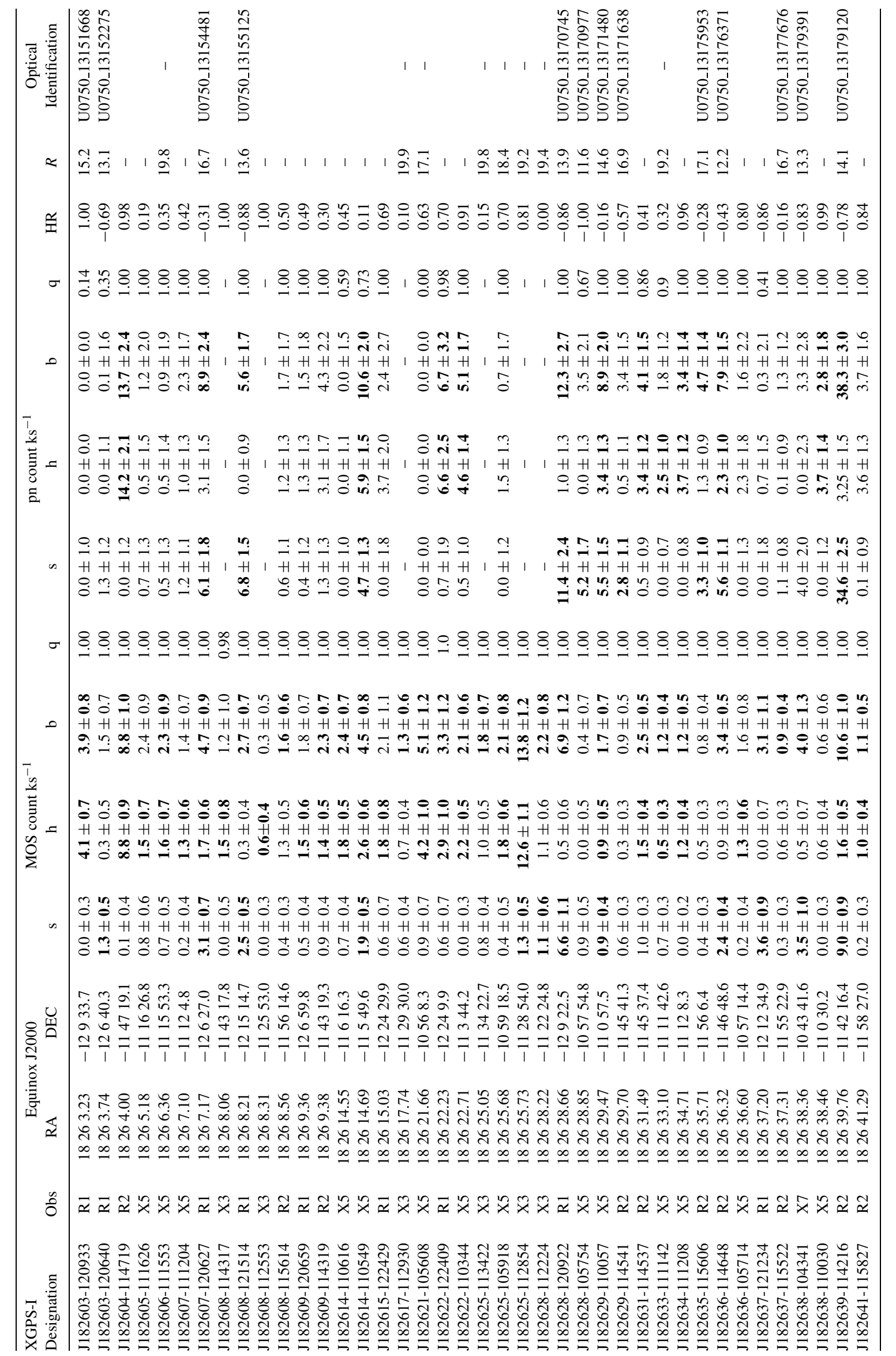




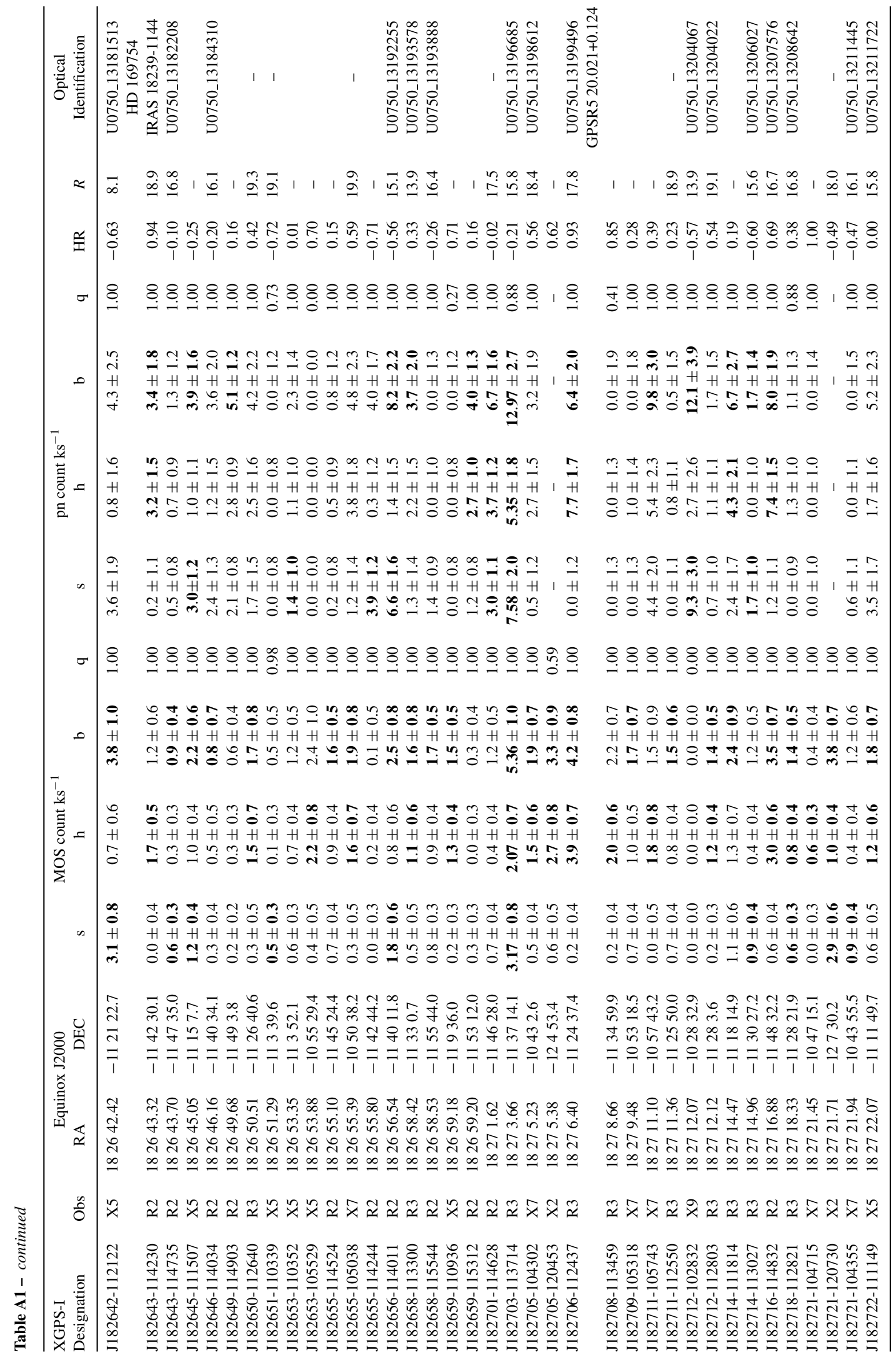




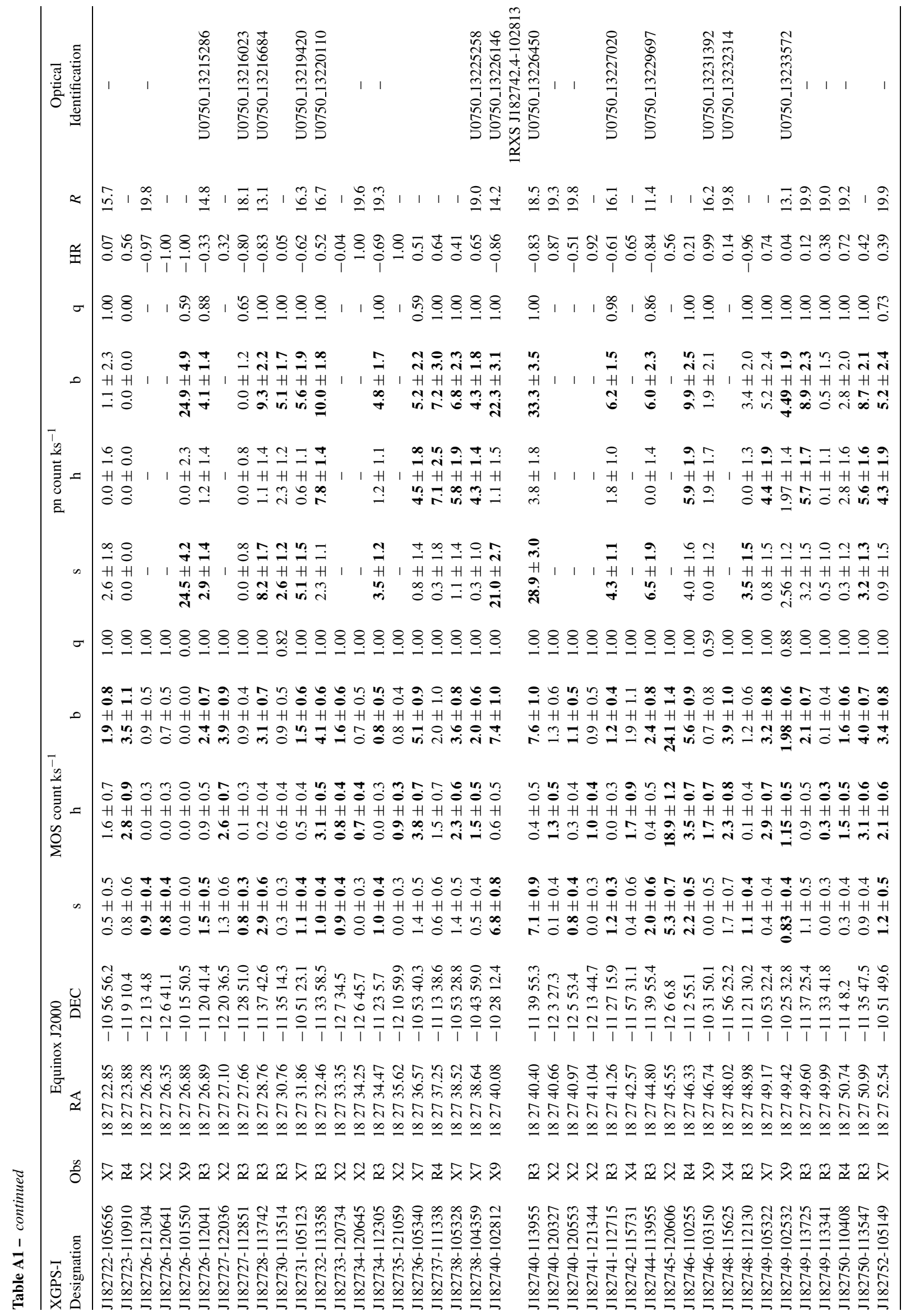




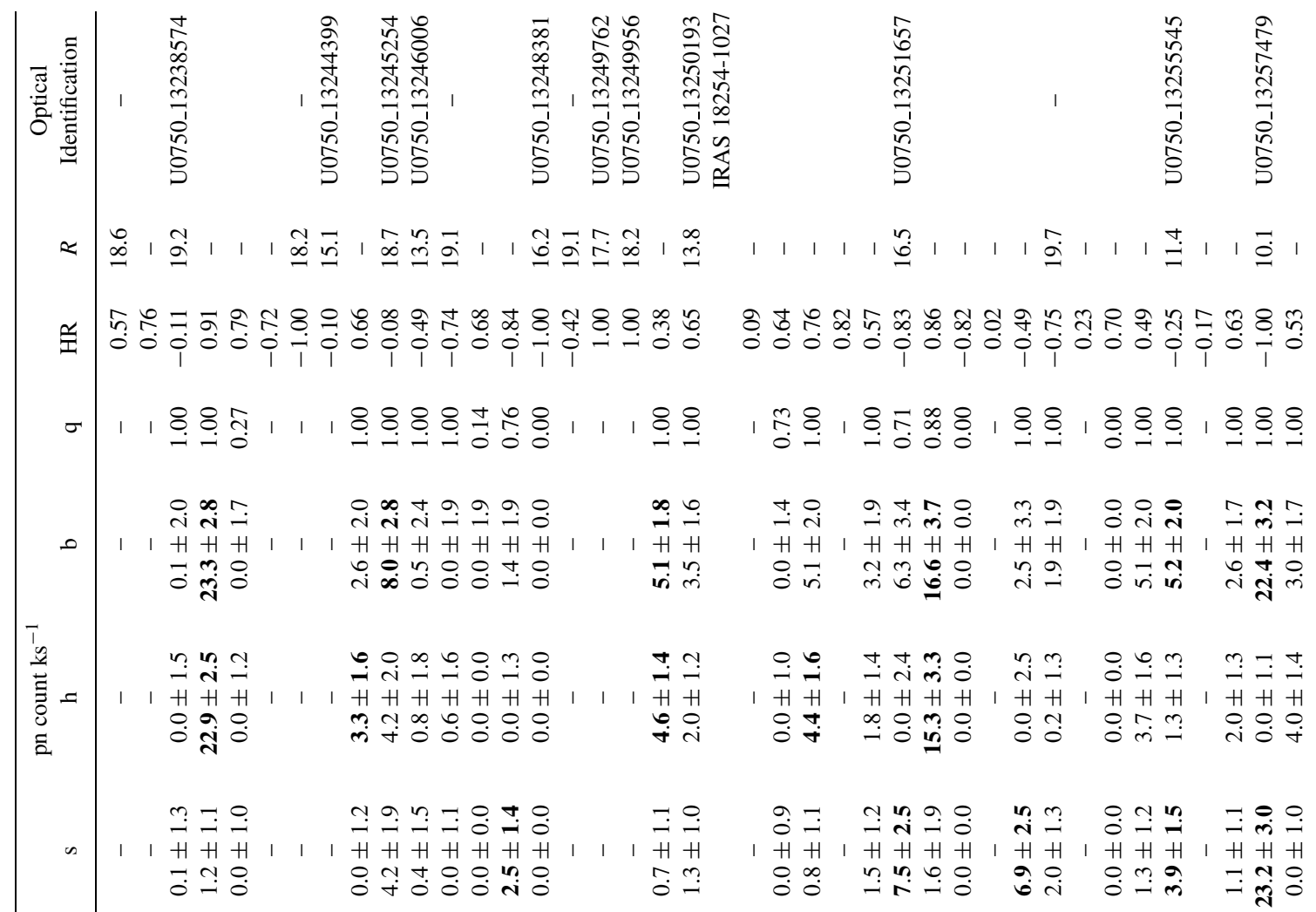

$\sigma$

8.8.8.8.8.8.8. \% 8.8.8

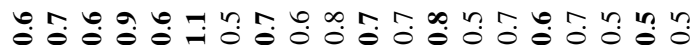

م $H+H+H+H+H+H+H+H+H+H+H+H+$

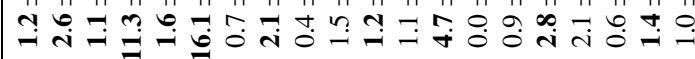

节 $0+H+H+H+H+H+H+H+H+H+H+H+H+H+H$ 品 $\sum$

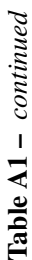

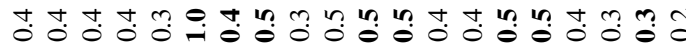
H H H H H H H H H H H H H H H H H H H

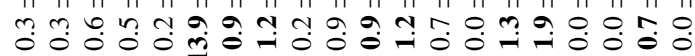

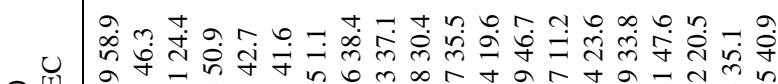

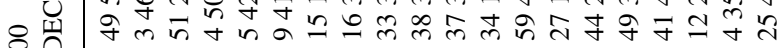

过

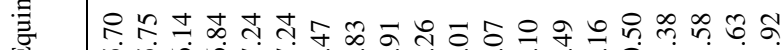

I « nि

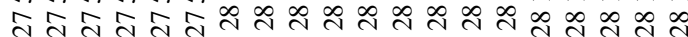

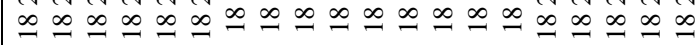

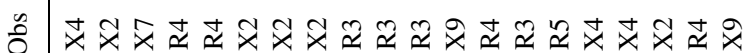

齐

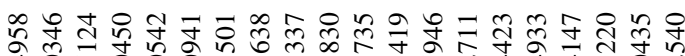

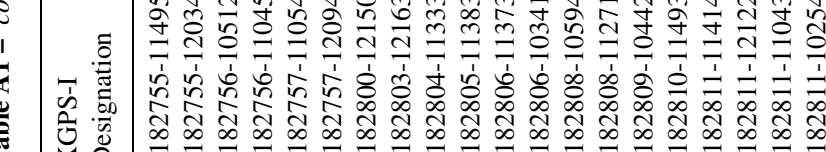

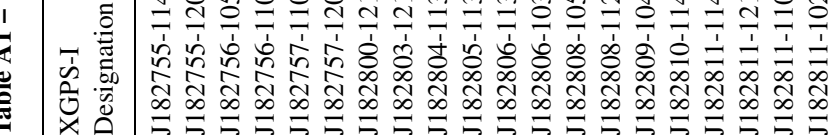

\& \& \& \& \& \& \& \& \& \& \& \& \& \&

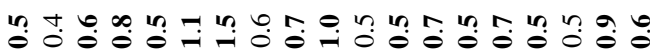

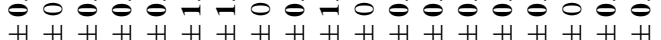

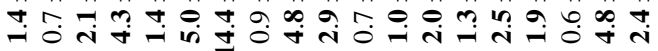

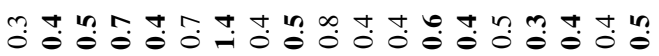
$H+H+H+H+H H H+H+H H+H+H+H+H$ 붕

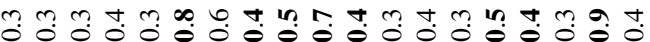
$+\mathrm{H}+\mathrm{H}+\mathrm{H}+\mathrm{H}+\mathrm{H}+\mathrm{H}+\mathrm{H}+\mathrm{H}+\mathrm{H}+\mathrm{H}+\mathrm{H}$

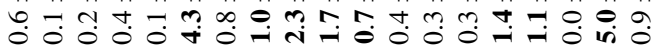

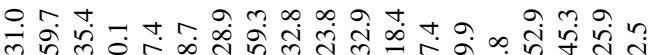
m $\infty$ m

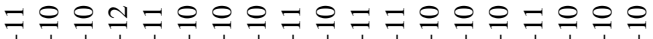

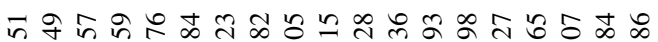

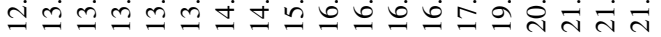
i

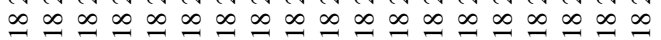

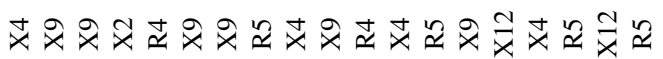

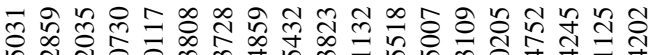

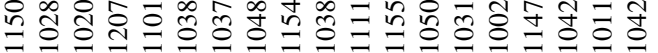

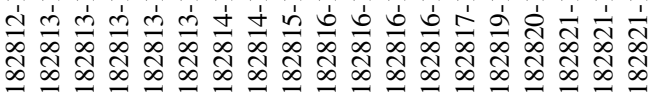




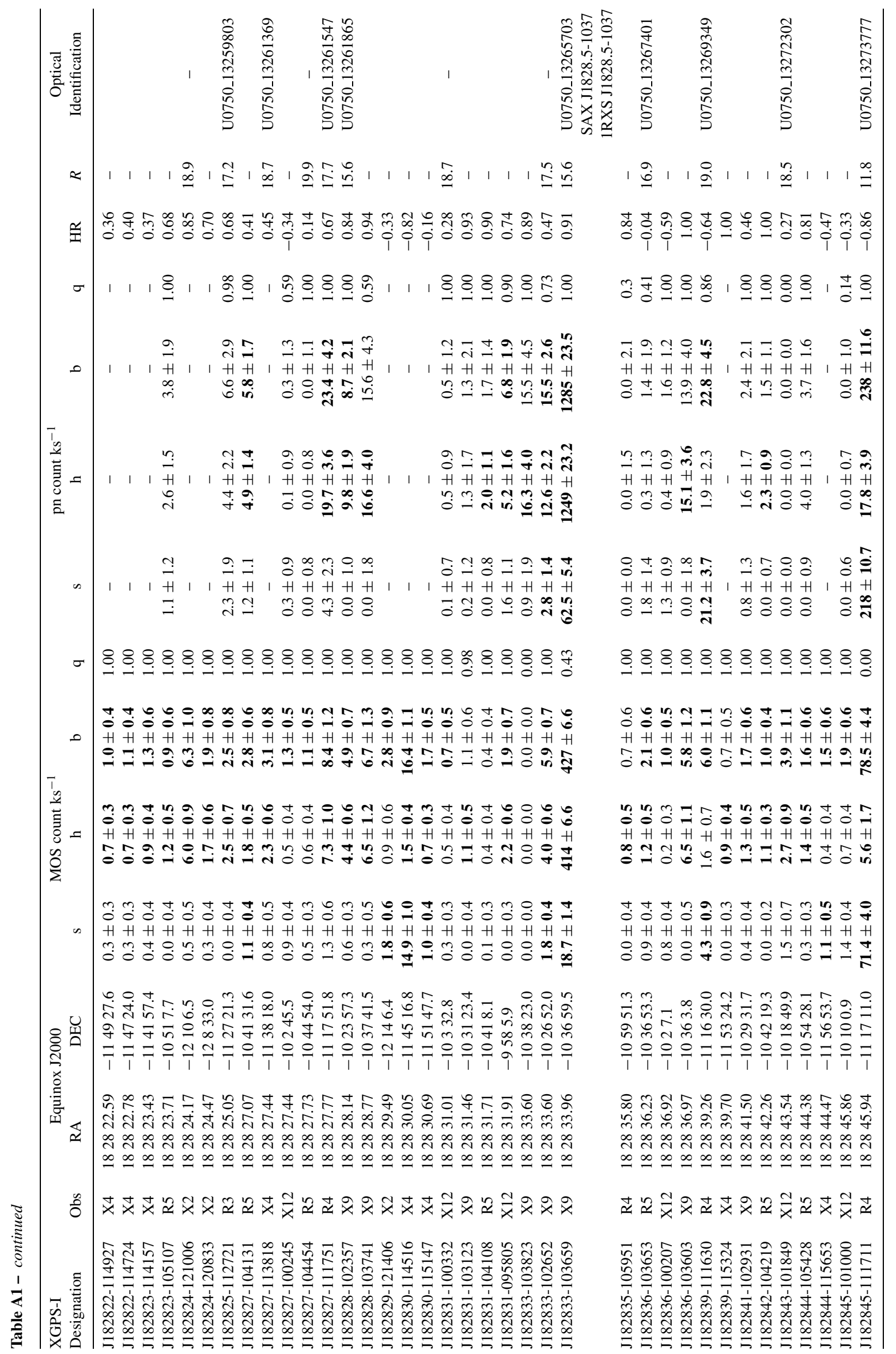




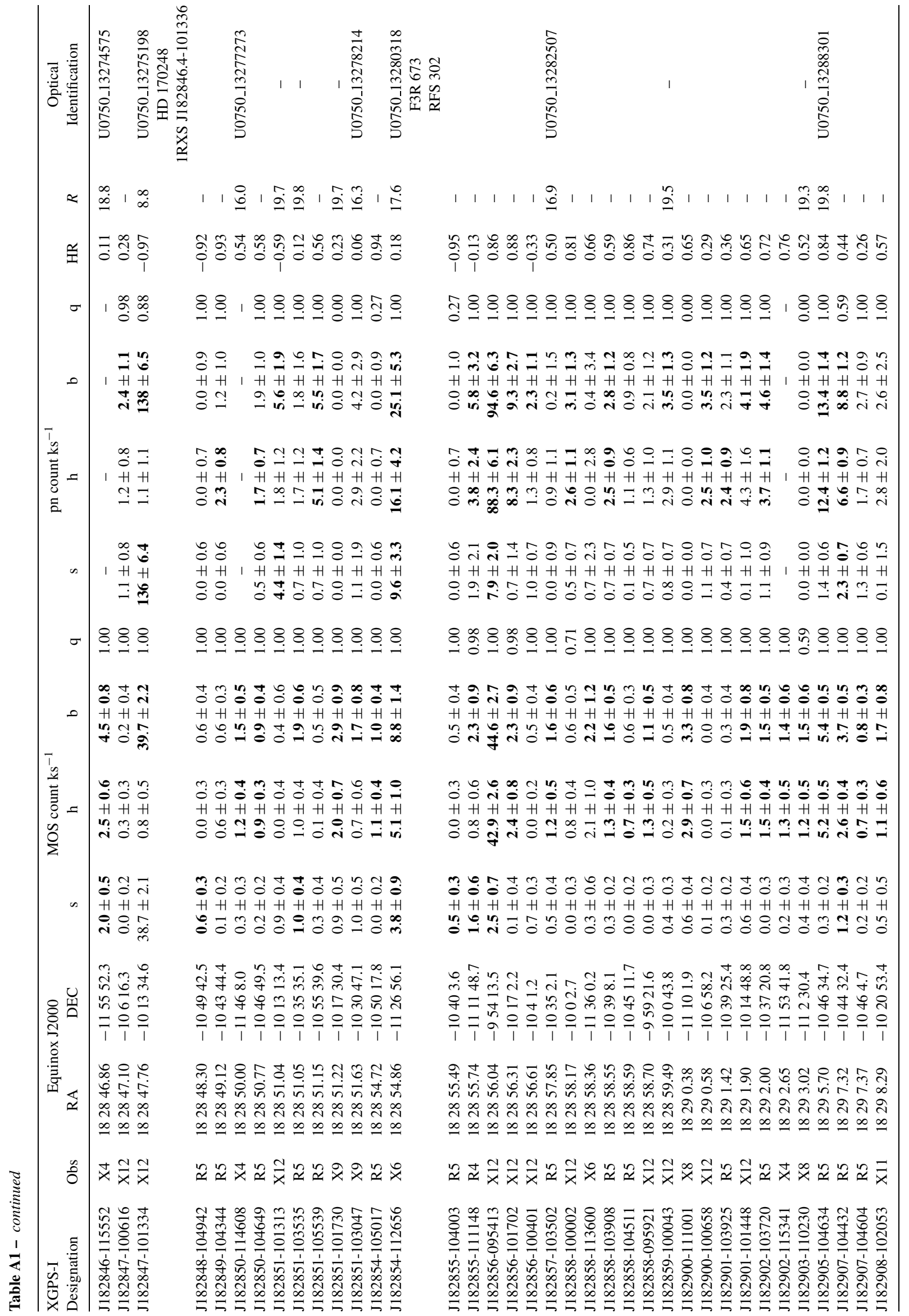




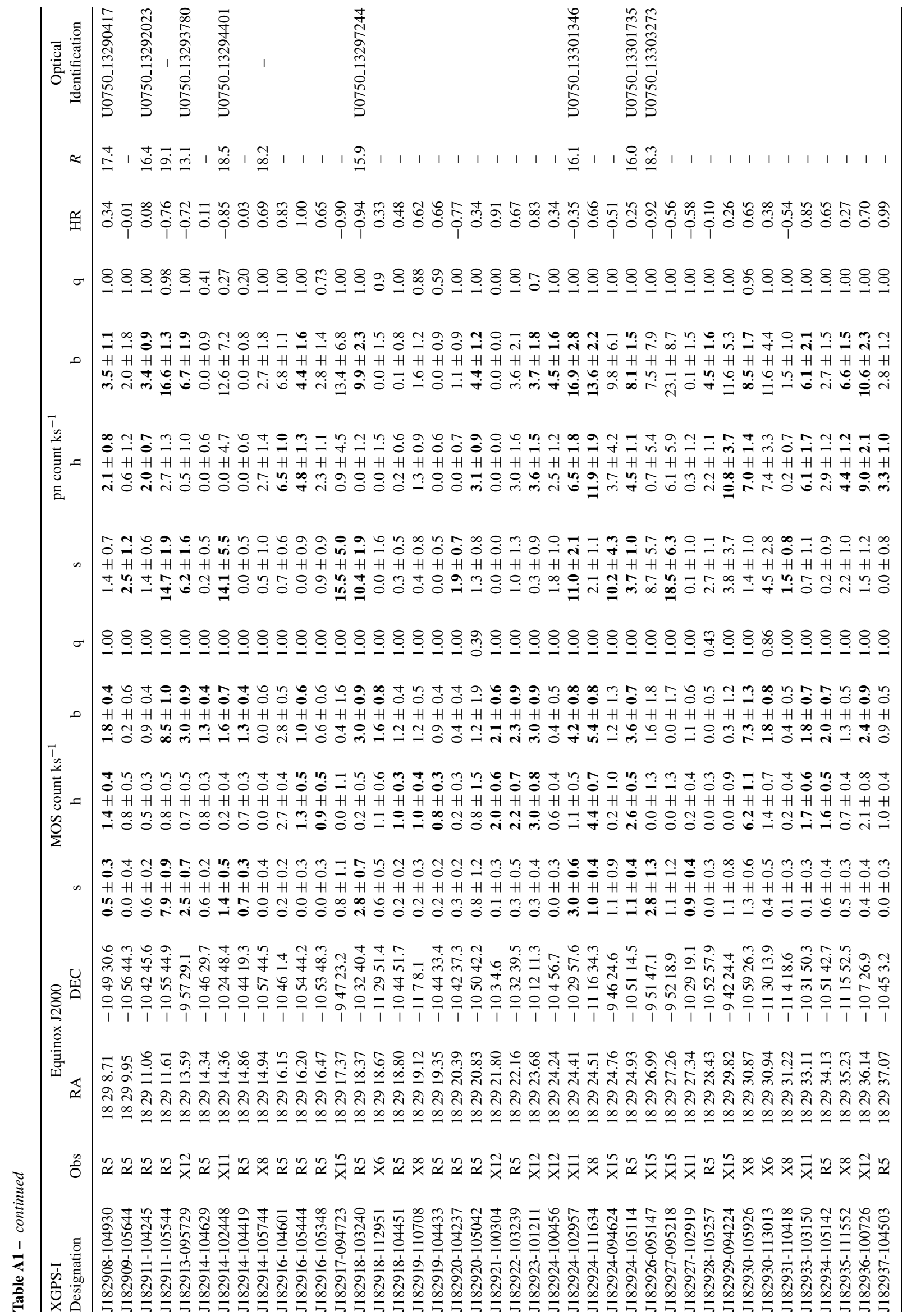




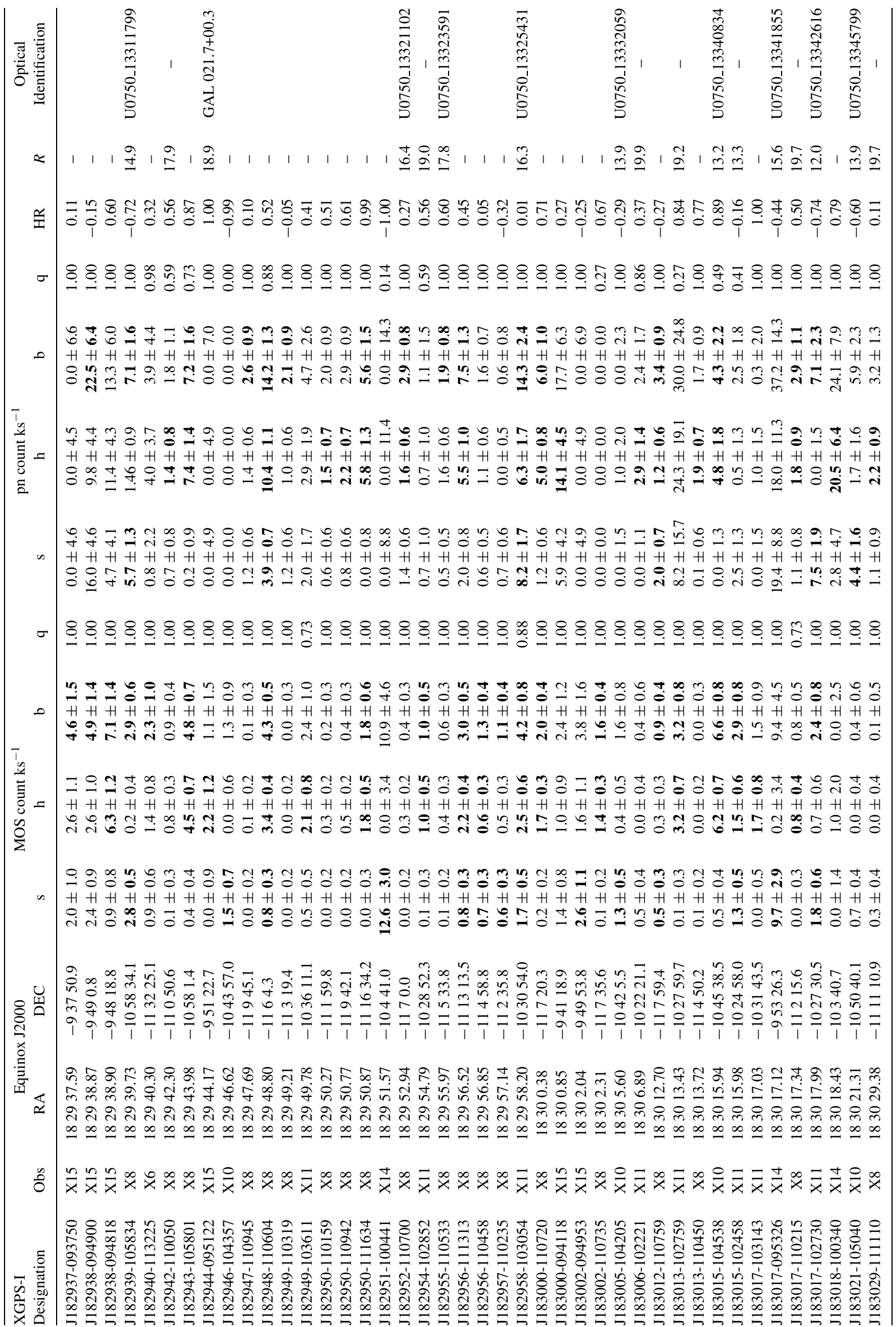




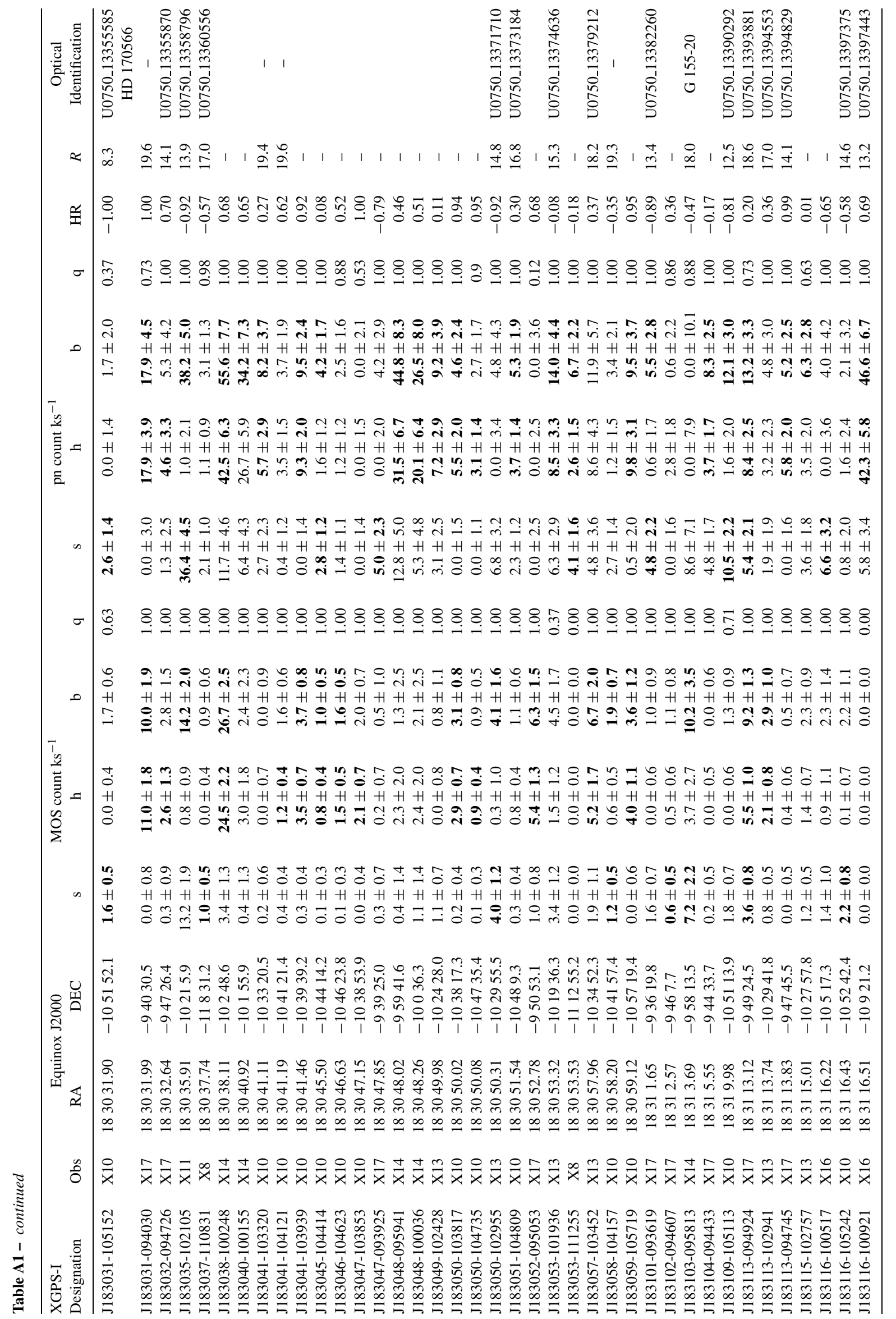




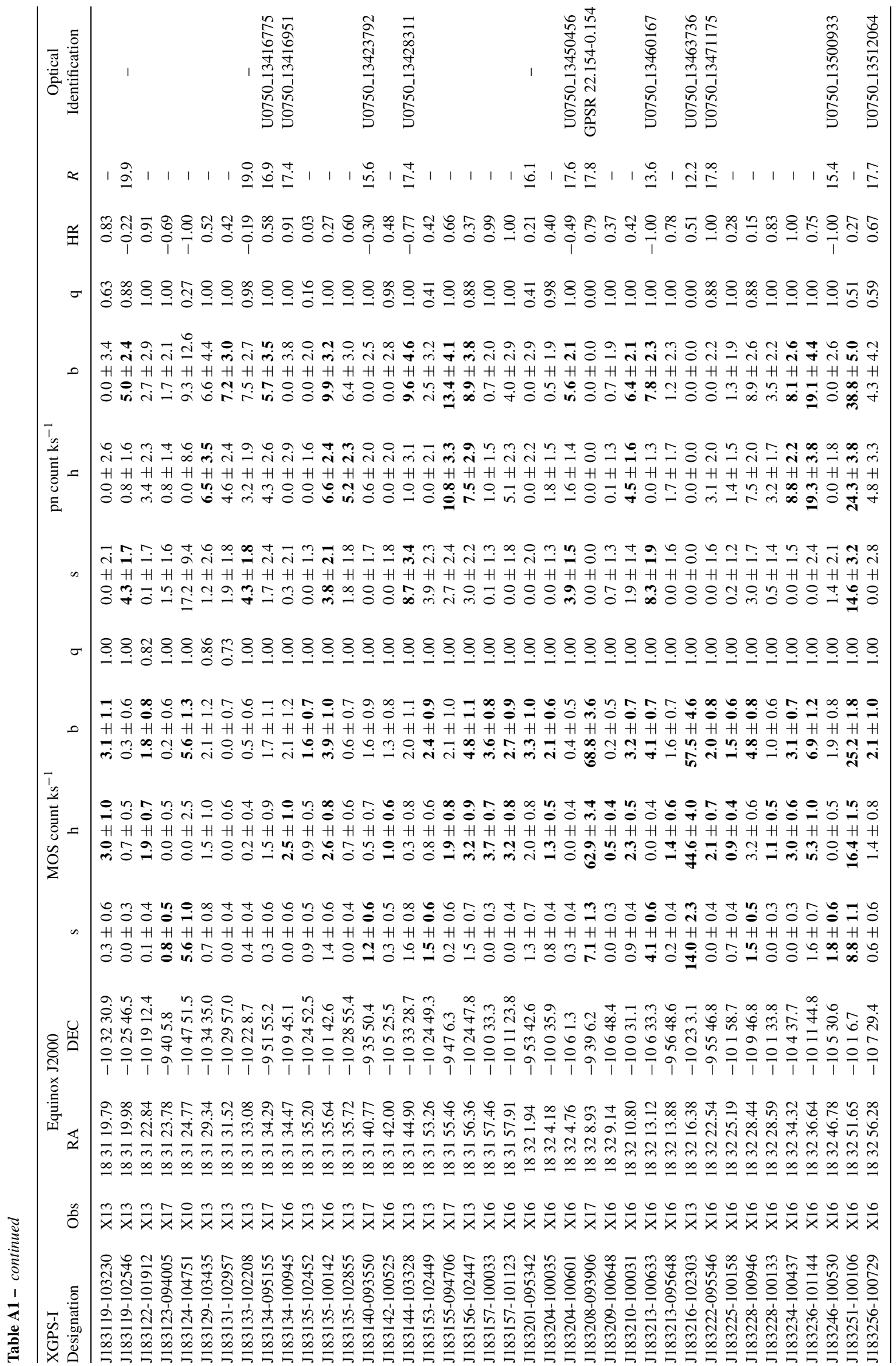

